ESAIM: PROCEEDINGS, December 2012, Vol. 38, p. 387-408

F. Coquel, M. Gutnic, P. Helluy, F. Lagoutière, C. Rohde, N. Seguin, Editors

\title{
A MULTISCALE METHOD FOR COMPRESSIBLE LIQUID-VAPOR FLOW WITH SURFACE TENSION*
}

\author{
Felix Jaegle ${ }^{1}$, Christian Rohde ${ }^{2}$ and Christoph Zeiler ${ }^{2}$
}

\begin{abstract}
Discontinuous Galerkin methods have become a powerful tool for approximating the solution of compressible flow problems. Their direct use for two-phase flow problems with phase transformation is not straightforward because this type of flows requires a detailed tracking of the phase front. We consider the fronts in this contribution as sharp interfaces and propose a novel multiscale approach. It combines an efficient high-order Discontinuous Galerkin solver for the computation in the bulk phases on the macro-scale with the use of a generalized Riemann solver on the micro-scale. The Riemann solver takes into account the effects of moderate surface tension via the curvature of the sharp interface as well as phase transformation.

First numerical experiments in three space dimensions underline the overall performance of the method.
\end{abstract}

\section{INTRODUCTION}

We consider an inviscid compressible fluid at constant temperature $\theta>0$ in an open bounded set $\Omega \subset \mathbb{R}^{d}$ with $d \in\{1,2,3\}$. It can appear either in a liquid or in a vapor phase. For any $t \in[0, T], T>0$, we assume that $\Omega$ splits up into the union of two open sets $\Omega_{\mathrm{vap}}(t), \Omega_{\text {liq }}(t)$, which contain the two bulk phases, and a hypersurface $\Gamma(t)$ that separates the two bulks. The hypersurface is called the sharp interface. In both bulk phases we assume that the dynamics of the fluid is governed by the isothermal Euler equations

$$
\begin{array}{ccc}
\varrho_{t}+\quad \operatorname{div}(\varrho \boldsymbol{v}) & = \\
(\varrho \boldsymbol{v})_{t}+\operatorname{div}(\varrho \boldsymbol{v} \otimes \boldsymbol{v}+p(\varrho) \boldsymbol{I}) & = & \mathbf{0}
\end{array} \quad \text { in }\left\{(\boldsymbol{x}, t) \in \Omega \times(0, T) \mid \boldsymbol{x} \in \Omega_{\mathrm{vap}}(t) \cup \Omega_{\mathrm{liq}}(t)\right\} .
$$

Here $\varrho=\varrho(\boldsymbol{x}, t)>0$ denotes the unknown density field and $\boldsymbol{v}=\boldsymbol{v}(\boldsymbol{x}, t)=\left(v_{1}(\boldsymbol{x}, t), \cdots, v_{d}(\boldsymbol{x}, t)\right)^{t} \in \mathbb{R}^{d}$ the unknown velocity field. The pressure $p=p(\varrho)$ is a given scalar function and $\boldsymbol{I} \in \mathbb{R}^{d \times d}$ the $d$-dimensional unit matrix. For later use we remark that the pressure is related to the Helmholtz free energy $\psi=\psi(\varrho)$ by

$$
p(\varrho)=\varrho^{2} \psi^{\prime}(\varrho)
$$

\footnotetext{
* The authors gratefully acknowledge the support by the German Research Foundation (DFG) through TRR 75 „Tropfendynamische Prozesse unter extremen Umgebungsbedingungen ", the grant RO 2222/4-1, the Cluster of Excellence in Simulation Technology (EXC 310/1) at the University of Stuttgart as well as the Carl Zeiss Foundation.

1 Institut für Aerodynamik and Gasdynamik, Universität Stuttgart, Pfaffenwaldring 21, 70569 Stuttgart, Germany; e-mail: Felix.Jaegle@iag.uni-stuttgart.de

${ }^{2}$ Institut für Angewandte Analysis und Numerische Simulation, Universität Stuttgart, Pfaffenwaldring 57, 70569 Stuttgart, Germany; e-mail: \{Christian.Rohde, Christoph.Zeiler\}@mathematik.uni-stuttgart.de
}

(C) EDP Sciences, SMAI 2012 


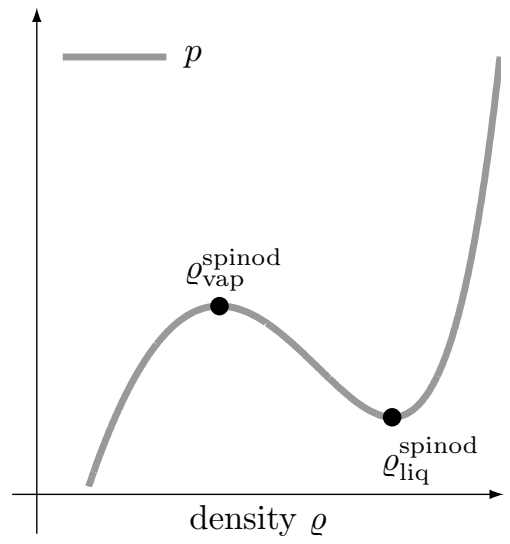

FiguRE 1. Van der Waals pressure function $p=p(\varrho)$.

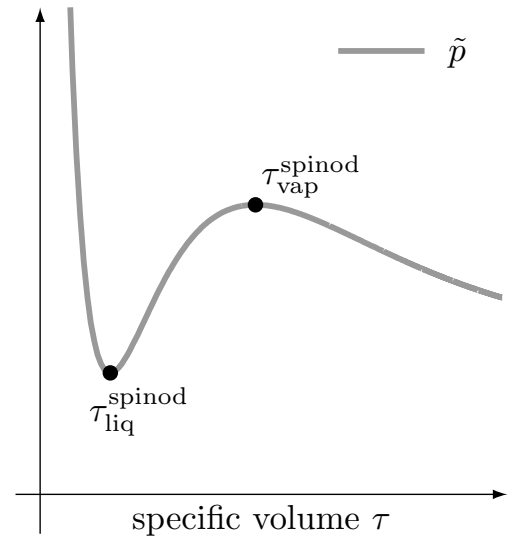

Figure 2. Van der Waals pressure function $\tilde{p}=\tilde{p}(\tau)$.

As a prototype example we consider here the van der Waals pressure

$$
p(\varrho)=\frac{R \theta \varrho}{1-b \varrho}-a \varrho^{2} \quad \text { for } \varrho \in\left(0, b^{-1}\right)
$$

with the specific choices

$$
\theta=0.85, \quad a=3, \quad b=1 / 3 \quad \text { and } \quad R=8 / 3 .
$$

From the graph in figure 1 we observe that $p$ is increasing in the intervals $\mathcal{A}_{\text {vap }}:=\left(0, \varrho_{\text {vap }}^{\text {spinod }}\right)$ and $\mathcal{A}_{\text {liq }}:=$ $\left(\varrho_{\text {liq }}^{\text {spinod }}, b\right)$ which define the vapor and the liquid phase. Note that $(1)$ is hyperbolic in both of these bulk phases and elliptic in the so-called spinodal region $\left(\varrho_{\mathrm{vap}}^{\text {spinod }}, \varrho_{\mathrm{liq}}^{\text {spinod }}\right)$, regardless of the velocity. Let us note that the bulk equations in (1) can be summarized for $\boldsymbol{U}=\left(\varrho, \varrho v_{1}, \ldots, \varrho v_{d}\right)^{t}$ in the conservation form

$$
\boldsymbol{U}_{t}+\boldsymbol{F}^{1}(\boldsymbol{U})_{x_{1}}+\cdots+\boldsymbol{F}^{d}(\boldsymbol{U})_{x_{d}}=\mathbf{0}
$$

with appropriately defined fluxes $\boldsymbol{F}^{1}, \ldots, \boldsymbol{F}^{d}$.

The phase boundary between liquid and vapor phase is represented by the (dynamic) sharp interface $\Gamma(t)$ for $t \in[0, T)$. For $\boldsymbol{\xi} \in \Gamma(t)$ let us denote the speed of $\Gamma(t)$ in the normal direction $\boldsymbol{n}=\boldsymbol{n}(\boldsymbol{\xi}, t) \in \mathcal{S}^{d-1}$ by $s=s(\boldsymbol{\xi}, t) \in \mathbb{R}$. The normal is always chosen as pointing into the vapor domain $\Omega_{\text {vap }}(t)$. Across the interface the following trace conditions have to be satisfied.

$$
\begin{aligned}
\{\{\varrho(\boldsymbol{v} \cdot \boldsymbol{n}-s)\} & =0, \\
\{\{\varrho(\boldsymbol{v} \cdot \boldsymbol{n}-s) \boldsymbol{v}+p(\varrho) \boldsymbol{n}\} & =(d-1) \gamma \kappa \boldsymbol{n} .
\end{aligned}
$$

Thereby $\{a\}\}:=a_{+}-a_{-}$and $a_{ \pm}=\lim _{\varepsilon \rightarrow 0} a(\boldsymbol{\xi} \pm \varepsilon \boldsymbol{n})$ for some quantity $a$ defined in $\Omega_{\mathrm{vap}}(t) \cup \Omega_{\mathrm{liq}}(t)$. In $(7)$ by $\kappa=\kappa(\boldsymbol{\xi}, t) \in \mathbb{R}$ we denote the mean curvature of $\Gamma(t)$ associated with orientation given through the choice of the normal $\boldsymbol{n}$. The surface tension coefficient is $\gamma>0$, assumed to be constant.

Condition (6) ensures the local conservation of mass across $\Gamma(t)$. The second relation (7) corresponds to a dynamical version of the Young-Laplace law for static phase boundaries. As we want to take into account phase transformation we have to provide an additional condition that controls the mass transfer and is consistent with the second law of thermodynamics. Our choice can be understood as an adaption of the Liu-criterion which is frequently used for real gas applications [17]. Details will be provided in Section 2. The full model (1), (6), (7) 
has to completed by the initial position $\Gamma(0)$ of the interface and the initial condition

$$
\boldsymbol{U}(\boldsymbol{x}, 0)=\boldsymbol{U}_{0}(\boldsymbol{x}) \quad \text { for } \quad \boldsymbol{x} \in \Omega,
$$

such that $\boldsymbol{U}_{0}(\boldsymbol{x}) \in \mathcal{A}_{\mathrm{liq} / \mathrm{vap}} \times \mathbb{R}^{d}$ for $\boldsymbol{x} \in \Omega_{\mathrm{liq} / \mathrm{vap}}$. As boundary conditions we use slip conditions in our numerical computations.

Up to our knowledge not so much is known analytically for $(1),(6),(7)$ in multiple space dimensions. Seminal well-posedness results for (viscous) two-phase flow without explicit phase transformation can be found in [20]. The stability of sharp interfaces in compressible flow (with a different treatment of the mass transfer) has been analyzed in [1].

The problem (1), (6), (7) has the form of a free-boundary problem that is particularly challenging for the numerical discretization. In this paper we will present a novel method for its efficient multi-dimensional numerical simulation, at least in the case of moderately curved interfaces. To handle curvature and phase transformation it turns out that a heterogeneous multiscale method (HMM) in the sense of [21] is an appropriate approach (cf. $[12,19]$ for related treatment of shock wave-like fronts). We consider the isothermal Euler equations in the bulk phases as the macro-scale model. To solve the macro-scale model we will use an implementation of the Discontinuous-Galerkin spectral element method (DGSEM) as developed in [14]. The effect of the trace conditions (6) and in particular the Young-Laplace equation (7) enters via a kind of micro-scale model ${ }^{1}$ which will be a generalized Riemann problem with non-homogeneous jump conditions. For the curvature-free case and using a completely different mass transfer via the interface this approach has been introduced in $[5,11,16]$. It relies on ghostfluid ideas tracing back to [7].

We describe now the basic elements of the HMM which also structure the rest of this paper. Assume that a triangulation of elements for $\Omega$ with mesh size parameter $h>0$ is given. In fact we will use a hexahedral mesh later on but the mechanism is independent of this choice. For the $n$th discrete time step $t=t^{n} \in(0, T)$ let us denote by $\Gamma_{h}^{n}$ an approximation of $\Gamma\left(t^{n}\right)$ that will be referred to as the computational interface. In the (sample) case $d=3$ it is supposed to consist of a family of mesh faces such that it separates uniquely two sets of elements $\Omega_{h \text {,vap }}^{n}, \Omega_{h \text {,liq }}^{n} \subset \Omega$. As in the continuous setting these sets are disjoint, associated with the discrete bulk phases, and supposed to satisfy $\Omega=\Omega_{h \text {,vap }}^{n} \cup \Omega_{h \text { liq }}^{n}$. For each element in $\Omega_{h \text {,vap }}^{n}\left(\Omega_{h \text {,liq }}^{n}\right)$ the discrete solution $\boldsymbol{U}_{\text {vap }}^{n}\left(\boldsymbol{U}_{\text {liq }}^{n}\right)$ is given by a fixed-order polynomial such that the density component is either in the vapor phase or in the liquid phase. We also consider a (level-set) function $\Phi_{h}: \Omega \rightarrow \mathbb{R}$ that defines a zero level-set $\Phi_{h}^{n}=\left\{\boldsymbol{x} \in \Omega \mid \Phi_{h}\left(\boldsymbol{x}, t^{n}\right)=0\right\}$. The zero level-set provides a second, in fact more accurate, approximation of $\Gamma\left(t^{n}\right)$.

Then we can summarize our algorithm to advance to the next time level as follows.

Algorithm 1.1 (Heterogeneous multiscale method).

Step 1 [Computation of discrete curvature] For each face $\mathcal{S} \in \Gamma_{h}^{n}$ an approximate local mean curvature value $\kappa(\mathcal{S})$ is computed.

Step 2 [Solution of a generalized Riemann problem (micro-scale solution)] For all numerical flux computations associated with some face $\mathcal{S} \in \Gamma_{h}^{n}$ (see Step 3 below) a Riemann problem is solved. The initial states of the Riemann problem are provided by values of the approximate solutions $\boldsymbol{U}_{\mathrm{liq}}^{n}$ in the associated element in $\Omega_{h, \text { liq }}^{n}$ and $\boldsymbol{U}_{\text {vap }}^{n}$ in the associated element in $\Omega_{h, \mathrm{vap}}^{n}$. The Riemann solver takes into account the local curvature $\kappa(\mathcal{S})$ from Step 1.

The Riemann solution contains a phase boundary connecting two states $\boldsymbol{U}_{\text {liq }}^{*}(\mathcal{S}) \in \mathcal{A}_{\text {liq }} \times \mathbb{R}, \boldsymbol{U}_{\text {vap }}^{*}(\mathcal{S}) \in$ $\mathcal{A}_{\text {vap }} \times \mathbb{R}$ and is moving with local speed $s(\mathcal{S})$.

Step 3 [Update of the bulk flow variables (macro-scale solution)] The explicit DGSEM is used to advance the density and velocity field in $\Omega_{h \text {,vap }}^{n}$ and $\Omega_{h, \text { liq }}^{n}$ to the next time level $t^{n+1}$. For the numerical

\footnotetext{
${ }^{1}$ The choice of the terms macro-/micro-scale should not be related here directly to specific spatial scales but is rather used to motivate our procedure.
} 
flux evaluation at the intersection of $\Omega_{h, \text { vap }}^{n}\left(\Omega_{h, \text { liq }}^{n}\right)$ and $\Gamma_{h}^{n}$ the state $\boldsymbol{U}_{\text {vap }}^{*}(\mathcal{S})\left(\boldsymbol{U}_{\text {liq }}^{*}(\mathcal{S})\right)$ from Step 2 is used. The time step is controlled by a CFL-like condition.

Step 4 [Update of the level-set function] The local normal front speeds $s(\mathcal{S})$ calculated in Step 2 for any face in $\Gamma_{h}^{n}$ are extended to a global velocity field $\boldsymbol{s}_{\mathrm{PB}}: \Omega \rightarrow \mathbb{R}^{d}$. The global velocity field is used to advance $\Phi_{h}^{n}$ to $\Phi_{h}^{n+1}$ (via solving a level-set equation, also with the DGSEM).

Step 5 [Reconstruction of a unique flow field and transport of the sharp interface] The zero level-set $\Phi_{h}^{n+1}$ determines new sets $\Omega_{h, \text { vap }}^{n+1}$ and $\Omega_{h, \text { liq }}^{n+1}$ and thus the new computational interface $\Gamma_{h}^{n+1}$. The bulk flow field will be extrapolated to the new geometry.

The first core element of the multiscale approach is the generalized Riemann solver on the micro-scale (Step 2). It is presented in Section 2. This micro-scale solver is only well-posed for moderate values of curvature (cf. [18] for a complete discussion and more general micro-scale solvers). The second core part is the Discontinuous Galerkin method used as the macro-scale solver (Step 3 and 4) that will be described in Section 3. Our implementation relies on the high-order DGSEM solver on hexahedral meshes as introduced in Step 3. In fact the presentation of the HMM above is simplified as -due to the high-order DGSEM solver- the Riemann solver must be activated for several integration points on the faces and for several Runge-Kutta steps used to achieve the same order in time. The steps 1, 4,5 are related to the transport of the interface and the transfer of information between scales. These tasks are challenging, especially for the case of two or three-dimensional flows. We will summarize the details in Section 4. In the final section we will present preliminary numerical results in three space dimensions. To end this introduction let us note that the overall HMM can handle arbitrary curvature. The restriction to small values is only due to the choice of the micro-scale solver. Algorithm 1.1 belongs to the class of sharpinterface methods, the phase boundary is not smeared out and the method does not introduce spurious density values.

\section{The Micro-Scale Solver: A Generalized Riemann Solution}

According to Step 2 in the HMM concept described in Section 1 the micro-scale solver provides an exact solution of a one-dimensional Riemann problem. For each element face quadrature point and in every Runge-Kutta time step the macro-scale solver assigns Riemann states $\boldsymbol{U}_{\text {liq }}=\left(\varrho_{\text {liq }}, v_{\text {liq }}\right)^{t} \in \mathcal{A}_{\text {liq }} \times \mathbb{R}$, $\boldsymbol{U}_{\text {vap }}:=\left(\varrho_{\text {vap }}, v_{\text {vap }}\right)^{t} \in \mathcal{A}_{\text {vap }} \times \mathbb{R}$ and a local curvature value $\kappa \in \mathbb{R}$ as input for the Riemann solver. The micro-scale solver gives back a phase boundary wave (i.e., states $\boldsymbol{U}_{\text {liq }}^{*} \in \mathcal{A}_{\text {liq }} \times \mathbb{R}, \boldsymbol{U}_{\text {vap }}^{*} \in \mathcal{A}_{\text {vap }} \times \mathbb{R}$ and speed $s \in \mathbb{R}$ ) that appears uniquely in the solution of the Riemann problem (cf. Theorem 2.6 below). Before we present the solver in detail let us fix the micro-scale problem.

For the sake of simplicity, we express the exact solution of the Riemann problem using Lagrangian coordinates. Naming Lagrangian coordinates by $(\xi, t)$, the unknowns are the specific volume $\tau=\tau(\xi, t)=1 / \varrho(\xi, t)$ and the fluid velocity $v=v(\xi, t)$, summarized in $\tilde{\boldsymbol{U}}=(\tau, v)^{t}$. As a function of $\tau$ the van der Waals pressure function (3) writes

$$
\tilde{p}(\tau)=p(1 / \tau)=\frac{R \theta}{\tau-b}-\frac{a}{\tau^{2}} \text { for } \tau \in(b, \infty)
$$

The corresponding sets of vapor and liquid specific volumes are denoted as $\tilde{\mathcal{A}}_{\text {liq }}:=\left(b, \tau_{\text {liq }}^{\text {spinod }}\right), \quad \tilde{\mathcal{A}}_{\text {vap }}:=$ $\left(\tau_{\text {vap }}^{\text {spinod }}, \infty\right)($ cf. figure 2$)$ and the complete admissible bulk set is

$$
\tilde{\mathcal{A}}:=\tilde{\mathcal{A}}_{\text {liq }} \cup \tilde{\mathcal{A}}_{\text {vap }}
$$

Then the generalized Riemann problem consists in finding a solution $\tilde{\boldsymbol{U}}(\xi, t)=(\tau(\xi, t), v(\xi, t))^{t} \in \tilde{\mathcal{A}} \times \mathbb{R}$ of

$$
\left(\begin{array}{l}
\tau \\
v
\end{array}\right)_{t}+\left(\begin{array}{c}
-v \\
\tilde{p}(\tau)
\end{array}\right)_{\xi}=\left(\begin{array}{l}
0 \\
0
\end{array}\right) \quad \text { with } \quad \tilde{\boldsymbol{U}}(\xi, 0)= \begin{cases}\tilde{\boldsymbol{U}}_{\text {liq }}:=\left(\tau_{\text {liq }}, v_{\text {liq }}\right)^{t} & \text { for } \xi<0, \\
\tilde{\boldsymbol{U}}_{\text {vap }}:=\left(\tau_{\text {vap }}, v_{\text {vap }}\right)^{t} & \text { for } \xi>0 .\end{cases}
$$




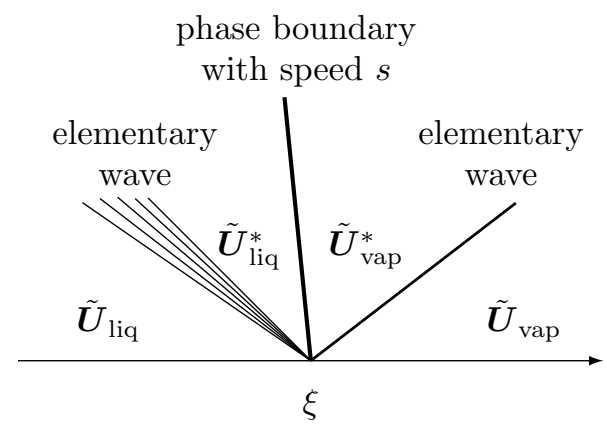

FIGURE 3. Sketch of the wave fan. One elementary wave in the liquid phase, followed by one intermediate phase boundary propagating with speed $s$ and one elementary wave in the vapor phase.

We seek for self-similar functions that match the following structure: one standard elementary wave (admissible shock wave, rarefaction wave or attached rarefaction-shock wave) in the liquid phase starting from $\tilde{\boldsymbol{U}}_{\text {liq }}$, one intermediate phase boundary, and one standard elementary wave in the vapor phase connecting to $\tilde{\boldsymbol{U}}_{\text {vap }}$. The phase boundary is a discontinuous wave connecting $\tilde{\boldsymbol{U}}_{\text {liq }}^{*}=\left(\tau_{\text {liq }}^{*}, v_{\text {liq }}^{*}\right)^{t} \in \tilde{\mathcal{A}}_{\text {liq }} \times \mathbb{R}, \tilde{\boldsymbol{U}}_{\text {vap }}^{*}=\left(\tau_{\text {vap }}^{*}, v_{\text {vap }}^{*}\right)^{t} \in \tilde{\mathcal{A}}_{\text {vap }} \times \mathbb{R}$ with speed $s \in \mathbb{R}$ such that the conditions (cf. (6), (7))

$$
\begin{aligned}
& s\left(\tau_{\text {vap }}^{*}-\tau_{\text {liq }}^{*}\right)+v_{\text {vap }}^{*}-v_{\text {liq }}^{*}=0, \\
& s\left(v_{\text {vap }}^{*}-v_{\text {liq }}^{*}\right)+\tilde{p}\left(\tau_{\text {vap }}^{*}\right)-\tilde{p}\left(\tau_{\text {liq }}^{*}\right)=(d-1) \gamma \kappa
\end{aligned}
$$

hold. For a sketch of the solution structure we refer to figure 3 .

The problem (10) is non-standard due to the non-homogeneous condition (12). Moreover and more fundamentally a well-posed solution can only be expected if an appropriate entropy condition is enforced. Both issues are clarified in the next subsections.

\subsection{Exact solution of the Riemann problem (10)}

In [8] the authors consider the Riemann problem (10) with $\kappa \equiv 0$. They substitute the non-monotone van der Waals function (9) by a non-increasing pressure isotherm using the so-called Maxwell construction. In [17] the authors follow the same concept for the full Euler system. In both cases the Liu criterion provides an appropriate selection criterion to enforce the unique solvability. We will generalize this concept towards the curvaturedependent situation $\kappa \neq 0$ and thereby transform condition (12) into a classical homogeneous Rankine-Hugoniot condition for the momentum equation with a new (non-increasing) pressure function $\tilde{p}^{\kappa}:(b, \infty) \rightarrow(0, \infty)$.

The construction procedure for $\tilde{p}^{\kappa}$ starts with modifications of the Helmholtz free energy. Transforming relation (2) gives $\tilde{p}(\tau)=-\tilde{\psi}^{\prime}(\tau)$ and thus with (9) the formula

$$
\tilde{\psi}(\tau):=\psi(1 / \tau)=-\frac{a}{\tau}-R \theta \ln (\tau-b) \quad \text { for } \tau \in(b, \infty)
$$

As the first step we define the auxiliary free energy function $\tilde{\psi}_{\text {aux }}^{\kappa}: \tilde{\mathcal{A}}_{\text {liq }} \cup \tilde{\mathcal{A}}_{\text {vap }} \rightarrow \mathbb{R}$ by adding a curvature term:

$$
\tilde{\psi}_{\text {aux }}^{\kappa}(\tau):= \begin{cases}\tilde{\psi}(\tau)-(d-1) \tau \gamma \kappa & \text { for } \tau \in \tilde{\mathcal{A}}_{\text {liq }}, \\ \tilde{\psi}(\tau) & \text { for } \tau \in \tilde{\mathcal{A}}_{\text {vap }}\end{cases}
$$

The function $\tilde{\psi}_{\text {aux }}^{\kappa}$ is convex in $\tilde{\mathcal{A}}_{\text {liq }}$ and in $\tilde{\mathcal{A}}_{\text {vap }}$. In the next step, we define the curvature dependent free energy function $\tilde{\psi}^{\kappa}:(b, \infty) \rightarrow \mathbb{R}$ as the convex envelope of the auxiliary function $\tilde{\psi}_{\text {aux }}^{\kappa}$. This can be understood as our 


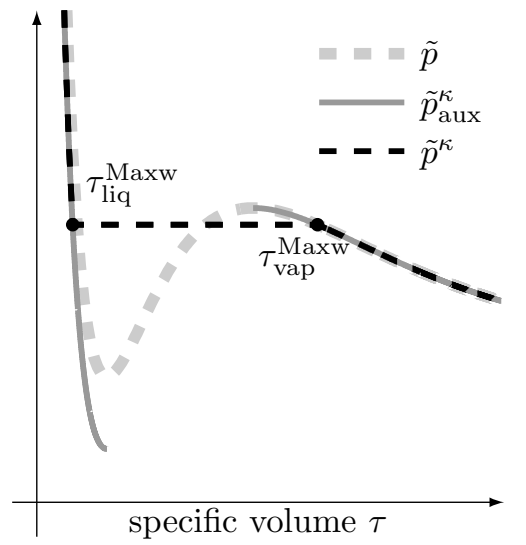

Figure 4. Pressure functions.

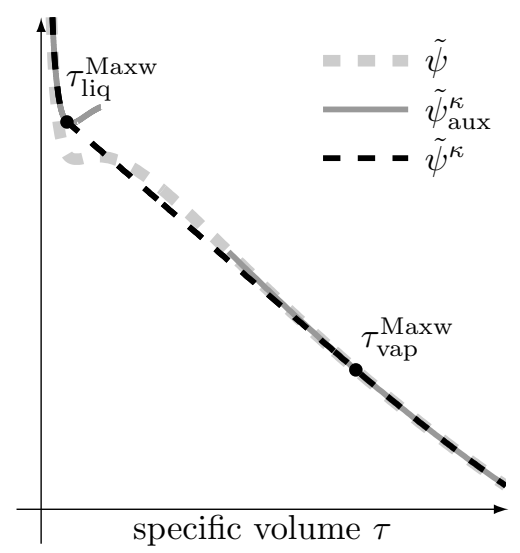

FIGURE 5. Free energy functions.

generalized Maxwell construction, and we call the end points of the line segment connecting $\left(\tau_{\text {liq }}^{\mathrm{Maxw}}, \tilde{\psi}^{\kappa}\left(\tau_{\mathrm{liq}}^{\mathrm{Maxw}}\right)\right)$ and $\left(\tau_{\text {vap }}^{\text {Maxw }}, \tilde{\psi}^{\kappa}\left(\tau_{\text {vap }}^{\text {Maxw }}\right)\right)$ generalized Maxwell points (cf. figure 5). For $\kappa=0$ it coincides with the classical Maxwell construction as used in [8]. Thus we have

$$
\tilde{\psi}^{\kappa}(\tau):= \begin{cases}\tilde{\psi}_{\text {aux }}^{\kappa}(\tau) & \text { for } \tau \in\left(b, \tau_{\text {liq }}^{\text {Maxw }}\right], \\ \tilde{\psi}_{\text {aux }}^{\kappa}\left(\tau_{\text {liq }}^{\text {Maxw }}\right)-\tau \tilde{p}^{\text {Maxw }} & \text { for } \tau \in\left(\tau_{\text {liq }}^{\text {Maxw }}, \tau_{\text {vap }}^{\text {Maxw }}\right], \\ \tilde{\psi}_{\text {aux }}^{\kappa}(\tau) & \text { for } \tau \in\left(\tau_{\text {vap }}^{\text {Maxw }}, \infty\right),\end{cases}
$$

where $\tilde{p}^{\text {Maxw }}$ is the generalized Maxwell pressure, defined through

$$
\tilde{p}^{\mathrm{Maxw}}:=\frac{\tilde{\psi}_{\text {aux }}^{\kappa}\left(\tau_{\text {vap }}^{\mathrm{Maxw}}\right)-\tilde{\psi}_{\text {aux }}^{\kappa}\left(\tau_{\text {liq }}^{\mathrm{Maxw}}\right)}{\tau_{\text {vap }}^{\mathrm{Maxw}}-\tau_{\text {liq }}^{\mathrm{Maxw}}} .
$$

Finally, we define the associated curvature dependent pressure function

$$
\tilde{p}^{\kappa}(\tau):= \begin{cases}\tilde{p}(\tau)+(d-1) \gamma \kappa & \text { for } \tau \in\left(b, \tau_{\text {liq }}^{\mathrm{Maxw}}\right], \\ \tilde{p}^{\mathrm{Maxw}} & \text { for } \tau \in\left(\tau_{\text {liq }}^{\mathrm{Maxw}}, \tau_{\text {vap }}^{\mathrm{Maxw}}\right], \\ \tilde{p}(\tau) & \text { for } \tau \in\left(\tau_{\text {vap }}^{\mathrm{Maxw}}, \infty\right),\end{cases}
$$

such that the thermodynamic relation $-\frac{\partial}{\partial \tau} \tilde{\psi}^{\kappa}(\tau)=\tilde{p}^{\kappa}(\tau)$ holds for all $\tau \in(b, \infty)$. Figures 4 and 5 illustrate the whole procedure. In figure 4 , the auxiliary pressure is $\tilde{p}_{\text {aux }}^{\kappa}:=-\frac{\partial}{\partial \tau} \tilde{\psi}_{\text {aux }}^{\kappa}$.

\section{Remark 2.1.}

(i) Note that the curvature term $(d-1) \gamma \kappa$ in (13) is constant for one generalized Riemann problem of Step 2 in Algorithm 1.1. Thus, for one micro-scale step the curvature is frozen.

(ii) With our construction procedure, one obtains a curvature dependent pressure function (13) with the curvature term in the liquid branch. Since the solution of (10) will depend only on derivatives of $\tilde{p}^{\kappa}$ one can alternatively modify the vapor branch without altering the final solution.

(iii) It can be easily checked that the construction of $\tilde{p}^{\mathrm{Maxw}}$ is well-posed (the generalized Maxwell points exist uniquely and $\tilde{p}^{\kappa}$ is continuous) provided that the absolute value of $\kappa$ is small enough. For sufficiently large curvature the construction in not possible anymore. In our computations we have not been faced with a situation where the construction fails. From the point of view of modeling we think that the failure for large curvature is not a consequence of the procedure but of the (unrealistic) 
properties of the van der Waals model, in particular the shape of $\tilde{p}$ close to the spinodal region, and the isothermality assumption.

In the following we assume that $\tilde{p}^{\kappa}:(b, \infty) \rightarrow(0, \infty)$ is properly defined. With the monotone pressure function $\tilde{p}^{\kappa}$ and for the given mean curvature $\kappa$ we re-state now the generalized Riemann problem (10).

Find a self-similar weak solution $\tilde{\boldsymbol{U}}(\xi, t)=(\tau, v)^{t} \in \tilde{\mathcal{A}} \times \mathbb{R}$ such that

$$
\left(\begin{array}{l}
\tau \\
v
\end{array}\right)_{t}+\left(\begin{array}{c}
-v \\
\tilde{p}^{\kappa}(\tau)
\end{array}\right)_{\xi}=\left(\begin{array}{l}
0 \\
0
\end{array}\right) \quad \text { with } \quad \tilde{\boldsymbol{U}}(\xi, 0)= \begin{cases}\left(\tau_{\text {liq }}, v_{\text {liq }}\right)^{t} & : \xi<0 \\
\left(\tau_{\text {vap }}, v_{\text {vap }}\right)^{t} & : \xi>0\end{cases}
$$

Note that (14) is now a weakly hyperbolic system. Indeed, the two eigenvalues of the Jacobian matrix of the flux $\left(-v, \tilde{p}^{\kappa}(\tau)\right)^{t}$ are

$$
\lambda_{1 / 2}(\tau, v)=\mp c(\tau) \quad \text { with } \quad c=c(\tau)=\sqrt{-\frac{\partial}{\partial \tau} \tilde{p}^{\kappa}(\tau)},
$$

and the eigenvectors are $r_{1 / 2}(\tau)=(1, \mp c(\tau))^{t}$. Thus (14) is hyperbolic for $\tau>0$ but $\tau \notin\left(\tau_{\text {liq }}^{\text {Maxw }}, \tau_{\text {vap }}^{\text {Maxw }}\right)$. In case of $\tau \in\left(\tau_{\text {liq }}^{\mathrm{Maxw}}, \tau_{\text {vap }}^{\mathrm{Maxw}}\right)$ the sound speed $c(\tau)$ becomes zero and the eigenvectors do not form a basis of $\mathbb{R}^{2}$ any more. This loss of hyperbolicity was already pointed out in $[3,8]$ and the references therein.

Nevertheless the Rankine-Hugoniot conditions for equation (14) coincide with the jump conditions (11) and (12) in case of a phase boundary. For planar phase boundaries $(\kappa \equiv 0)$, Godlewski and Seguin showed in [8] that the weakly hyperbolic Riemann problem (14) together with the entropy criterion of Liu [15] is well-posed. Following exactly this approach, we can select a unique solution for curved phase boundaries, i.e. the Riemann problem (14).

The solution consists of rarefaction waves, discontinuous shock waves, attached waves and so-called phase boundaries. These waves are connected via intermediate states such that Liu's criterion is satisfied. In the following, we have a closer look at the solution.

Definition 2.2 (Shock Wave and Phase Boundary). A given left state $\tilde{\boldsymbol{U}}_{\mathrm{L}}=\left(\tau_{\mathrm{L}}, v_{\mathrm{L}}\right)^{t} \in \tilde{\mathcal{A}} \times \mathbb{R}$ and a right state $\tilde{\boldsymbol{U}}_{\mathrm{R}}=\left(\tau_{\mathrm{R}}, v_{\mathrm{R}}\right)^{t} \in \tilde{\mathcal{A}} \times \mathbb{R}$ are connected by a 1-shock wave (resp. 2-shock wave) with propagation speed $s=s\left(\tau_{\mathrm{L}}, \tau_{\mathrm{R}}\right)<0$ (resp. $\left.s>0\right)$ if

$$
\tilde{\boldsymbol{U}}(\xi, t)= \begin{cases}\tilde{\boldsymbol{U}}_{\mathrm{L}}, & \text { for } \xi-s t<0 \\ \tilde{\boldsymbol{U}}_{\mathrm{R}}, & \text { for } \xi-s t>0\end{cases}
$$

is a weak solution of system (14). We call the discontinuity wave $\tilde{\boldsymbol{U}}$ a phase boundary if $\tau_{\mathrm{L}} \in \tilde{\mathcal{A}}_{\text {liq }}$ and $\tau_{\mathrm{R}} \in \tilde{\mathcal{A}}_{\text {vap }}$. Here the speed $s=s\left(\tau_{\mathrm{L}}, \tau_{\mathrm{R}}\right)$ is given by

$$
s\left(\tau_{\mathrm{L}}, \tau_{\mathrm{R}}\right)= \pm \sqrt{-\frac{\tilde{p}^{\kappa}\left(\tau_{\mathrm{R}}\right)-\tilde{p}^{\kappa}\left(\tau_{\mathrm{L}}\right)}{\tau_{\mathrm{R}}-\tau_{\mathrm{L}}}} .
$$

Note that static phase boundaries $(s=0)$ require the generalized Maxwell states as left and right volume values (with $v_{\mathrm{L}}=v_{\mathrm{R}}$ ).

Definition 2.3 (Rarefaction Wave). Let a left state $\tilde{\boldsymbol{U}}_{\mathrm{L}}=\left(\tau_{\mathrm{L}}, v_{\mathrm{R}}\right)^{t} \in \tilde{\mathcal{A}} \times \mathbb{R}$ and right state $\tilde{\boldsymbol{U}}_{\mathrm{R}}=\left(\tau_{\mathrm{R}}, v_{\mathrm{L}}\right)^{t} \in$ $\tilde{\mathcal{A}} \times \mathbb{R}$ be given. Then

- $\tilde{\boldsymbol{U}}_{\mathrm{L}}$ and $\tilde{\boldsymbol{U}}_{\mathrm{R}}$ are connected by a 1-rarefaction wave if $\tau_{\mathrm{L}}<\tau_{\mathrm{R}}, \tilde{p}^{\kappa}$ is convex on $\left[\tau_{\mathrm{L}}, \tau_{\mathrm{R}}\right]$ and $v_{\mathrm{R}}=v_{\mathrm{L}}+H\left(\tau_{\mathrm{L}}, \tau_{\mathrm{R}}\right)$ holds,

- $\tilde{\boldsymbol{U}}_{\mathrm{L}}$ and $\tilde{\boldsymbol{U}}_{\mathrm{R}}$ are connected by a 2 -rarefaction wave if $\tau_{\mathrm{L}}>\tau_{\mathrm{R}}, \tilde{p}^{\kappa}$ is concave on $\left[\tau_{\mathrm{R}}, \tau_{\mathrm{L}}\right]$ and $v_{\mathrm{R}}=v_{\mathrm{L}}-H\left(\tau_{\mathrm{L}}, \tau_{\mathrm{R}}\right)$ holds. 

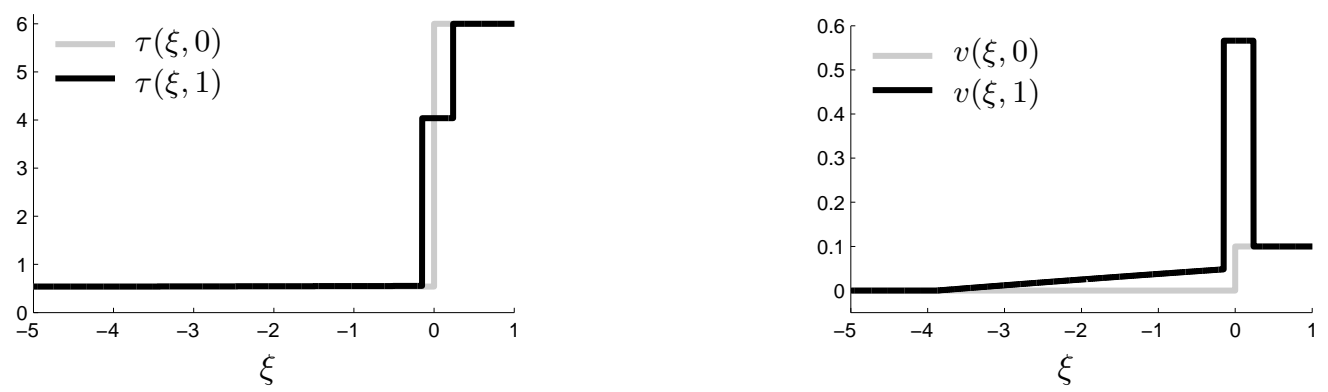

FiguRE 6. The left-hand graph shows the specific volume and the right-hand graph the velocity: gray lines indicate the initial condition, black lines the solution at $t=1$.

Here

$$
H\left(\tau_{\mathrm{L}}, \tau_{\mathrm{R}}\right):=\int_{I\left(\tau_{\mathrm{L}}, \tau_{\mathrm{R}}\right)} \sqrt{-\frac{\partial}{\partial \tau} \tilde{p}^{\kappa}(\tau)} \mathrm{d} \tau
$$

and $I\left(\tau_{\mathrm{L}}, \tau_{\mathrm{R}}\right):=\left(\min \left\{\tau_{\mathrm{L}}, \tau_{\mathrm{R}}\right\}, \max \left\{\tau_{\mathrm{L}}, \tau_{\mathrm{R}}\right\}\right)$.

We single out admissible discontinuity waves by applying Liu's criterion [15]. The criterion together with the Rankine-Hugoniot condition determines the solution of the generalized Riemann problem.

Definition 2.4 (Liu's Entropy Criterion). Any shock wave connecting a state $\tilde{\boldsymbol{U}}_{\mathrm{L}}=\left(\tau_{\mathrm{L}}, v_{\mathrm{L}}\right)^{t}$ and a state $\tilde{\boldsymbol{U}}_{\mathrm{R}}=\left(\tau_{\mathrm{R}}, v_{\mathrm{R}}\right)^{t}$ is said to fulfill Liu's entropy criterion if and only if

$$
s\left(\tau_{\mathrm{L}}, \tau_{\mathrm{R}}\right) \leq s\left(\tau_{\mathrm{L}}, \tau\right) \text { for all } \tau \in I\left(\tau_{\mathrm{L}}, \tau_{\mathrm{R}}\right),
$$

with $s$ as in (15).

An important consequence of this definition that relates Liu's criterion to the second law of thermodynamics has been proved in [15].

Lemma 2.5. Any shock wave that fulfills the Liu's entropy criterion satisfies

$$
s\left\{\left\{E^{\kappa}(\tau, v)\right\}-\left\{\left\{F^{\kappa}(\tau, v)\right\} \leq 0\right.\right.
$$

with entropy $E^{\kappa}(\tau, v):=\frac{1}{2} v^{2}+\tilde{\psi}^{\kappa}(\tau)$ and entropy flux $F^{\kappa}(\tau, v):=v \tilde{p}^{\kappa}(\tau)$ for all $\tau \in \tilde{\mathcal{A}}, v \in \mathbb{R}$.

Note that entropy $E^{\kappa}$ and entropy flux $F^{\kappa}$ in Lemma 2.5 are defined using the modified free energy. For a shock wave that connects states in the same phase the entropy/entropy flux is unchanged from the one-phase case since then $p$. However, for a phase boundary the entropy depends explicitly on the curvature. This is to be expected to account for the interfacial energy contribution (cf. [9] for a mathematical treatment in the static case).

Motivated by Lemma 2.5 we call any weak solution of (14) that satisfies (17) for all shock waves and phase boundaries an entropy solution of (10). Our construction leads to the following well-posedness result which is proved in [18] using monotonicity arguments.

Theorem 2.6. The Riemann problem (10) with (11), (12) has a unique entropy solution $\tilde{\boldsymbol{U}}=\tilde{\boldsymbol{U}}(\xi, t) \in \tilde{\mathcal{A}} \times \mathbb{R}$. The solution is self-similar and consists of exactly one phase boundary connecting intermediate states $\tilde{\boldsymbol{U}}_{\text {liq }}^{*}, \tilde{\boldsymbol{U}}_{\text {vap }}^{*}$ of different phases. One elementary wave connects the states $\tilde{\boldsymbol{U}}_{\text {liq }}, \tilde{\boldsymbol{U}}_{\text {liq }}^{*}$ in the liquid phase and one elementary wave the states $\tilde{\boldsymbol{U}}_{\mathrm{vap}}^{*}, \tilde{\boldsymbol{U}}_{\text {vap }}$ in the vapor phase. Elementary waves are either rarefaction waves, admissible shock waves or attached rarefaction-shock waves. For a sketch of such a wave fan see figure 3. 


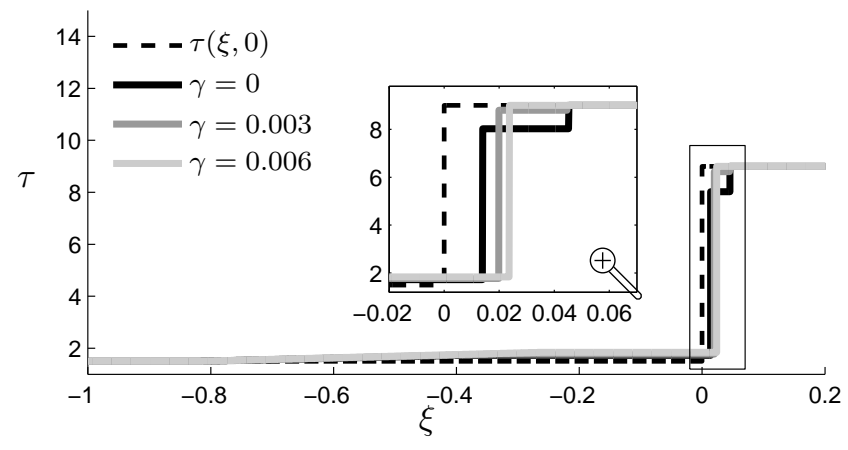

FiguRE 7. Specific volume distributions for different surface tension. The initial condition is given as dashed lines.

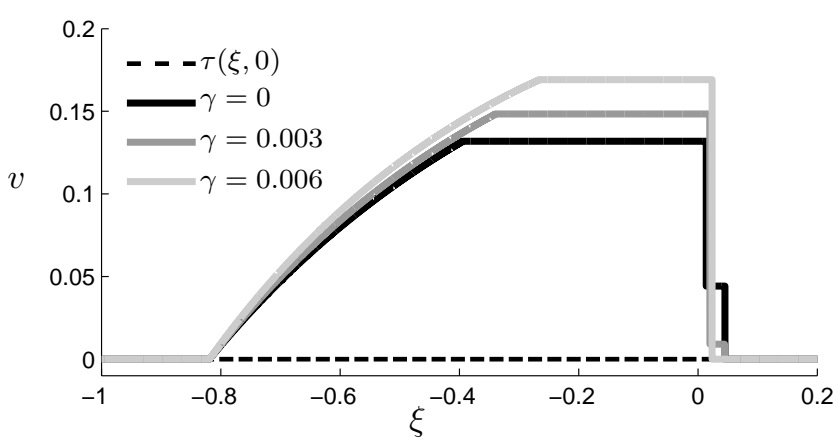

FIGURE 8. Velocity distributions for different surface tension. The initial condition is given as dashed lines.

Remark 2.7. Stationary solutions appear as interesting special cases of Theorem 2.6. The solution consists then of a single phase boundary. It is worthwhile to note that our solution reproduces the stationary YoungLaplace law, and moreover the chemical potential $\tilde{\mu}:=\tilde{\psi}(\tau)+\tau \tilde{p}(\tau)$ remains continuous across the phase phase boundary as is expected [9].

\subsection{Illustrating Examples}

We present here two wave pattern that comply with the solution described in Theorem 2.6.

Example 2.8 (A sample wave fan). Consider the initial condition

$$
\tilde{\boldsymbol{U}}(\xi, 0)= \begin{cases}\tilde{\boldsymbol{U}}_{\text {liq }}:=(1.8,0.05)^{t} & \text { for } \xi<0, \\ \tilde{\boldsymbol{U}}_{\text {vap }}:=(18,0)^{t} & \text { for } \xi>0,\end{cases}
$$

parameters for the van der Waals equation of state as in (4), $d=3, \kappa=-0.5$, and $\gamma=0.001$. Figure 6 shows the entropy solution and figure 3 a sketch of the corresponding wave fan. In this example, there are intermediate states $\tilde{\boldsymbol{U}}_{\text {liq }}^{*}$ and $\tilde{\boldsymbol{U}}_{\text {vap }}^{*}$ such that

- $\tilde{\boldsymbol{U}}_{\text {liq }}$ and $\tilde{\boldsymbol{U}}_{\text {liq }}^{*}$ are connected by a 1-rarefaction wave,

- $\tilde{\boldsymbol{U}}_{\text {liq }}^{*}$ and $\tilde{\boldsymbol{U}}_{\text {vap }}^{*}$ are connected by a phase boundary and

- $\tilde{\boldsymbol{U}}_{\text {vap }}^{*}$ and $\tilde{\boldsymbol{U}}_{\text {vap }}$ are connected by a 2 -shock wave.

Example 2.9 (Wave fans for different curvature values). Finally, we compare solutions for different curvature terms. Initial conditions are

$$
\tilde{\boldsymbol{U}}(\xi, 0)= \begin{cases}(1.8,0)^{t} & \text { for } \xi<0, \\ (9,0)^{t} & \text { for } \xi>0 .\end{cases}
$$

The surface tension coefficient $\gamma$ is taken from the set $\{0,0.003,0.006\}, d=3, \kappa=0.5$, and the parameters for the van der Waals equation of state as in (4). Figure 7 shows the specific volumes and figure 8 velocity distributions. One can interpret this example as considering spherical bubbles of the same radius in different fluids, i.e. different surface tension coefficients $\gamma$. As $\gamma$ increases the bubble tends to expand faster to release the interfacial energy. 


\section{The Macro-Scale Solver: a Discontinuous Galerkin spectral element METHOD}

In this section we describe the the macro-scale solver used in Step 3 and Step 4 of Algorithm 1.1. In both cases we rely on the Discontinuous Galerkin spectral element method (DGSEM).

\subsection{The Discontinuous Galerkin spectral element method for the 3D Euler equations}

Since our main focus is not the DGSEM scheme used in this study but the formulation of the multiscale algorithm we will restrict ourselves to the features important for the multiscale modeling. For more details, we refer to $[13,14]$. In view of our application we assume $d=3$, and $\Omega$ to be a cubic domain. Then, $\Omega$ is partitioned into a Cartesian mesh $\mathcal{T}=\left\{T_{\boldsymbol{i}} \mid \boldsymbol{i}=\left(i_{1}, i_{2}, i_{3}\right)^{t} \in \mathcal{I}:=\left\{1, \ldots, N^{1}\right\} \times\left\{1, \ldots, N^{2}\right\} \times\left\{1, \ldots, N^{3}\right\}\right\}$ of non-overlapping hexahedral elements given by $T_{\boldsymbol{i}}=\left(x_{i_{1}-1 / 2}, x_{i_{1}+1 / 2}\right) \times\left(x_{i_{2}-1 / 2}, x_{i_{2}+1 / 2}\right) \times\left(x_{i_{3}-1 / 2}, x_{i_{3}+1 / 2}\right)$. We define the directional element diameters

$$
\Delta x_{i_{k}}=x_{i_{k}+1 / 2}-x_{i_{k}-1 / 2}, \quad k \in\{1,2,3\}
$$

and the element center $\boldsymbol{x}_{\boldsymbol{i}}=\left(x_{i_{1}+1 / 2}+x_{i_{1}-1 / 2}, x_{i_{2}+1 / 2}+x_{i_{2}-1 / 2}, x_{i_{3}+1 / 2}+x_{i_{3}-1 / 2}\right)^{t} / 2$. Furthermore we define the set of neighbour indices by

$$
\mathcal{N}(i)=\left\{i \pm e_{1}, i \pm e_{2}, i \pm e_{3}\right\},
$$

where $\boldsymbol{e}_{k}, k=1,2,3$, denotes the $k$ th unit vector in $\mathbb{R}^{3}$. Note that $\mathcal{N}(\boldsymbol{i})$ might contain indices which do not belong to $\mathcal{I}$, these correspond to elements $T_{\boldsymbol{i}}$ such that $\partial T_{\boldsymbol{i}} \cap \partial \Omega \neq \emptyset$.

All computations will be done on the reference cube element $E:=(-1,1)^{3}$, cf. figure 9 . For each $i \in \mathcal{I}$ the affine transformation mapping $\Psi_{i}: E \rightarrow T_{i}$ is given through

$$
\Psi_{i}(\boldsymbol{\zeta})=\left(\frac{\Delta x_{i_{1}}}{2} \zeta_{1}, \frac{\Delta x_{i_{2}}}{2} \zeta_{2}, \frac{\Delta x_{i_{3}}}{2} \zeta_{3}\right)^{t}+\boldsymbol{x}_{\boldsymbol{i}}, \quad \boldsymbol{\zeta}=\left(\zeta_{1}, \zeta_{2}, \zeta_{3}\right)^{t}
$$

We have to specify element faces. For $\boldsymbol{i} \in \mathcal{I}$ and $\boldsymbol{j} \in \mathcal{N}(\boldsymbol{i}) \cap \mathcal{I}$ define $S_{\boldsymbol{i} \boldsymbol{j}}=\bar{T}_{\boldsymbol{i}} \cap \bar{T}_{\boldsymbol{j}}$. Otherwise, if $\mathcal{N}(\boldsymbol{i}) \cap\left(\mathbb{Z}^{3} \backslash \mathcal{I}\right) \neq \emptyset$ the set $\partial T_{\boldsymbol{i}} \cap \partial \Omega$ is not empty. Some index $\boldsymbol{j}$ in this set we can represent in the form $\boldsymbol{j}=\boldsymbol{i} \pm \boldsymbol{e}_{k}$. The set $S_{\boldsymbol{i} \boldsymbol{j}}$ is then that face of $T_{\boldsymbol{i}}$ such that the outer normal of $S_{\boldsymbol{i} \boldsymbol{j}}$ as subset of $\partial \Omega$ and $\pm \boldsymbol{e}_{k}$ point in the same direction. If we then denote the faces of $E$ naturally by $E_{k}$, let $k(\boldsymbol{i}, \boldsymbol{j}) \in\{ \pm 1, \pm 2, \pm 3\}$ be the unique index of the face of $E$ such that $\Psi\left(E_{k(i, j)}\right)=S_{\boldsymbol{i} \boldsymbol{j}}$, cf. figure 9 .

We consider now the bulk equations (5) and denote for each $\boldsymbol{i} \in \mathcal{I}$ by $\overline{\boldsymbol{U}}_{\boldsymbol{i}}(\boldsymbol{\zeta}, t):=\boldsymbol{U}\left(\Psi_{\boldsymbol{i}}(\boldsymbol{\zeta}), t\right)$ with $\Psi_{\boldsymbol{i}}(\boldsymbol{\zeta}) \in T_{\boldsymbol{i}}$ the transformed vector of unknowns in $E$. Then $\overline{\boldsymbol{U}}_{\boldsymbol{i}}$ is supposed to satisfy the transformed system

$$
\overline{\boldsymbol{U}}_{\boldsymbol{i}, t}+\mathcal{F}_{\boldsymbol{i}}^{1}\left(\overline{\boldsymbol{U}}_{\boldsymbol{i}}\right)_{\zeta_{1}}+\mathcal{F}_{\boldsymbol{i}}^{2}\left(\overline{\boldsymbol{U}}_{\boldsymbol{i}}\right)_{\zeta_{2}}+\mathcal{F}_{\boldsymbol{i}}^{3}\left(\overline{\boldsymbol{U}}_{\boldsymbol{i}}\right)_{\zeta_{3}}=\mathbf{0} \quad \text { in } E \times(0, T)
$$

In view of (5) the fluxes in (18) are given through $\mathcal{F}_{\boldsymbol{i}}^{k}=\frac{2}{\Delta x_{i_{k}}} \boldsymbol{F}^{k}, k=1,2,3$.

For directional polynomial degree $P \in \mathbb{N}$ and $\mathcal{P}=\{0, \ldots, P\}^{3}$ we consider now the tensor-product like approximation

$$
\overline{\boldsymbol{U}}_{\boldsymbol{i}}^{\Delta x}(\boldsymbol{\zeta}, t)=\sum_{\boldsymbol{p} \in \mathcal{P}} \hat{\boldsymbol{U}}_{\boldsymbol{i}, \boldsymbol{p}}(t) \psi_{\boldsymbol{p}}(\boldsymbol{\zeta}), \quad \psi_{\boldsymbol{p}}(\boldsymbol{\zeta})=l_{p_{1}}\left(\zeta_{1}\right) l_{p_{2}}\left(\zeta_{2}\right) l_{p_{3}}\left(\zeta_{3}\right)
$$

where $l_{p}=l_{p}(\zeta), p \in\{0, \ldots, P\}$, are the one-dimensional Lagrange polynomials of degree $P$ defined as

$$
l_{p}(\zeta)=\prod_{\substack{j=0 \\ j \neq p}}^{P} \frac{\zeta-\zeta^{j}}{\zeta^{p}-\zeta^{j}} .
$$




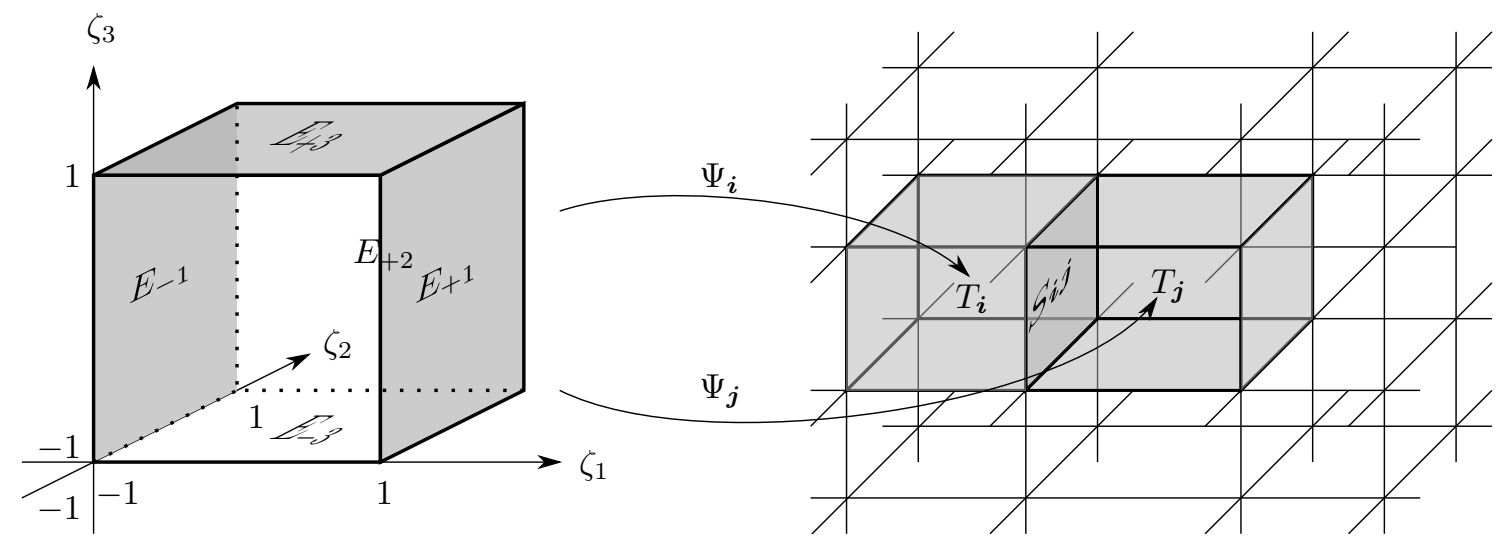

Figure 9. Left: reference element $E=(-1,1)^{3}$ and corresponding faces $E_{ \pm 1}, E_{+2}$ and $E_{ \pm 3}$. Right: exemplary elements $T_{\boldsymbol{i}}, T_{\boldsymbol{j}} \subset \Omega$ with common face $S_{\boldsymbol{i} \boldsymbol{j}}$. Corresponding affine transformation mappings $\Psi_{\boldsymbol{i}}, \Psi_{\boldsymbol{j}}$ from the reference space to the domain.

The pairwise distinct numbers $\zeta^{0}, \ldots, \zeta^{P} \in(-1,1)$ in $(20)$ are fixed below. Introducing now the elementindependent space

we observe $\overline{\boldsymbol{U}}_{\boldsymbol{i}}^{\Delta x}(\cdot, t) \in \mathcal{V}^{P}$.

$$
\mathcal{V}^{P}=\left[\operatorname{span}\left\{\psi_{\boldsymbol{p}} \mid \boldsymbol{p} \in \mathcal{P}\right\}\right]^{4}
$$

Furthermore we introduce some interpolation operators. The volume operator $I^{P}:\left(\mathrm{C}^{0}(E)\right)^{4} \rightarrow \mathcal{V}^{P}$ is uniquely defined in a way such that for $\boldsymbol{G} \in\left(\mathrm{C}^{0}(E)\right)^{4}$ the interpolation property

$$
I^{P}[\boldsymbol{G}]\left(\zeta^{p_{1}}, \zeta^{p_{2}}, \zeta^{p_{3}}\right)=\boldsymbol{G}\left(\zeta^{p_{1}}, \zeta^{p_{2}}, \zeta^{p_{3}}\right)
$$

holds for all $\boldsymbol{p} \in \mathcal{P}$.

For $k \in\{ \pm 1, \pm 2, \pm 3\}$ the surface operator $I_{k}^{P}:\left(\mathrm{C}^{0}\left(E_{k}\right)\right)^{4} \times\left(\mathrm{C}^{0}\left(E_{-k}\right)\right)^{4} \rightarrow \mathcal{V}_{k}^{P}$ with $\mathcal{V}_{k}^{P}=\left\{\Psi=\Psi(\zeta) \mid \zeta_{k}=\right.$ $\left.\operatorname{sgn}(k), \Psi \in \mathcal{V}^{P}\right\}$ is uniquely defined in the following way. For e.g. $k=+1(k=-1, \pm 2, \pm 3$ analogously $)$ and some $\boldsymbol{G} \in\left(\mathrm{C}^{0}\left(E_{k}\right)\right)^{4} \times\left(\mathrm{C}^{0}\left(E_{-k}\right)\right)^{4}$ the interpolation property

$$
I_{+1}^{P}[\boldsymbol{G}]\left(+1, \zeta^{p_{2}}, \zeta^{p_{3}}\right)=\boldsymbol{G}\left(+1, \zeta^{p_{2}}, \zeta^{p_{3}},-1, \zeta^{p_{2}}, \zeta^{p_{3}}\right)
$$

holds for all $\boldsymbol{p} \in \mathcal{P}$.

For the DGSEM scheme (in the integral formulation) we search now for a function $\boldsymbol{U}^{\Delta x}: \Omega \times[0, T] \rightarrow$ $\left(\mathcal{A}_{\text {liq }} \cup \mathcal{A}_{\text {vap }}\right) \times \mathbb{R}^{3}$ with

$$
\boldsymbol{U}^{\Delta x}(\boldsymbol{x}, t)=\overline{\boldsymbol{U}}_{\boldsymbol{i}}^{\Delta x}\left(\Psi_{\boldsymbol{i}}^{-1}(\boldsymbol{x}), t\right) \text { for }(\boldsymbol{x}, t) \in T_{\boldsymbol{i}} \times(0, T]
$$

such that

$$
\begin{aligned}
\frac{\partial}{\partial t} \int_{E} \overline{\boldsymbol{U}}_{\boldsymbol{i}}^{\Delta x} \psi_{\boldsymbol{q}} \mathrm{d} \boldsymbol{\zeta} & +\sum_{\boldsymbol{j} \in \mathcal{N}(\boldsymbol{i})} \int_{E_{k(\boldsymbol{i}, \boldsymbol{j})}} \mathcal{F}_{\boldsymbol{i}}^{*, k(\boldsymbol{i}, \boldsymbol{j})}\left(\overline{\boldsymbol{U}}_{\boldsymbol{i}}^{\Delta x}, \overline{\boldsymbol{U}}_{\boldsymbol{j}}^{\Delta x}\right) \psi_{\boldsymbol{q}} \mathrm{d} \boldsymbol{S} \\
& =\int_{E}\left(\mathcal{F}_{\boldsymbol{i}}^{1}\left(\overline{\boldsymbol{U}}_{\boldsymbol{i}}^{\Delta x}\right) \psi_{\boldsymbol{q}, \zeta_{1}}+\mathcal{F}_{\boldsymbol{i}}^{2}\left(\overline{\boldsymbol{U}}_{\boldsymbol{i}}^{\Delta x}\right) \psi_{\boldsymbol{q}, \zeta_{2}}+\mathcal{F}_{\boldsymbol{i}}^{3}\left(\overline{\boldsymbol{U}}_{\boldsymbol{i}}^{\Delta x}\right) \psi_{\boldsymbol{q}, \zeta_{3}}\right) \mathrm{d} \boldsymbol{\zeta} .
\end{aligned}
$$

holds for all $\boldsymbol{i} \in \mathcal{I}$, all $\boldsymbol{q} \in \mathcal{P}$ and $t \in(0, T]$. For $t=0$ the approximate function $\boldsymbol{U}^{\Delta x}(\cdot, 0)$ is obtained by transforming $I^{P}\left[\boldsymbol{U}_{0}\left(\Psi_{\boldsymbol{i}}\right)\right]$ into $\Omega$ for $\boldsymbol{i} \in \mathcal{I}$. Thereby $\boldsymbol{U}_{0}$ denotes the initial data from (8). The function $\mathcal{F}_{\boldsymbol{i}}^{*, k}:\left(\left(\mathcal{A}_{\text {liq }} \cup \mathcal{A}_{\text {vap }}\right) \times \mathbb{R}^{3}\right)^{2} \rightarrow \mathbb{R}^{4}$ is a numerical flux that is consistent with the oriented flux $\operatorname{sgn}(k) \mathcal{F}_{i}^{|k|}$ for 
$k \in\{ \pm 1, \pm 2, \pm 3\}$. In the computations of Section 5 we use the HLLC flux [6]. Note that the arguments $\overline{\boldsymbol{U}}_{\boldsymbol{i}}^{\Delta x}$ and $\overline{\boldsymbol{U}}_{\boldsymbol{j}}^{\Delta x}$ in the numerical flux have to be evaluated as one sided traces on $E_{k(\boldsymbol{i}, \boldsymbol{j})}$ for $\overline{\boldsymbol{U}}_{\boldsymbol{i}}^{\Delta x}$ and $E_{-k(\boldsymbol{i}, \boldsymbol{j})}$ for $\overline{\boldsymbol{U}}_{\boldsymbol{j}}^{\Delta x}$. For elements $T_{\boldsymbol{i}}$ with boundary surfaces $E_{\boldsymbol{i} \boldsymbol{j}}$ (i.e. $\boldsymbol{j} \in \mathcal{N}(\boldsymbol{i}) \cap\left(\mathbb{Z}^{3} \backslash \mathcal{I}\right)$ ) boundary fluxes have to be specified. In the applications we use slip boundary conditions. This means that $\overline{\boldsymbol{U}}_{\boldsymbol{j}}^{\Delta x}$ is chosen such that $\bar{\varrho}_{j}^{\Delta x}=\bar{\varrho}_{\boldsymbol{i}}^{\Delta x}$. The velocity components are chosen such that the tangential (normal) component is equal to the tangential (negative normal) component of $\overline{\boldsymbol{U}}_{\boldsymbol{i}}^{\Delta x}$.

The integral form (21) of the DGSEM scheme is not yet fully discrete as we have to specify the points $\zeta^{0}, \ldots, \zeta^{P} \in(-1,1)$, the quadrature formulas, and a time marching method.

Following the ideas of the DGSEM method [14] we choose $\zeta^{0}, \ldots, \zeta^{P}$ as the zeros of the $(P+1)$ th order Legendre polynomial which are located in $(-1,1)$. Then, the $(P$ th order) Gauss quadrature formula to approximate the one-dimensional integral $\int_{-1}^{1} g(\zeta) \mathrm{d} \zeta$ for $g:[-1,1] \rightarrow \mathbb{R}$ given by

$$
Q^{P}[g]=\sum_{p=0}^{P} \omega_{p} g\left(\zeta^{p}\right)
$$

is exact for polynomials up to order $2 P+1$. In $(22)$ the numbers $\omega_{0}, \ldots, \omega_{P}>0$ are the quadrature weights. Note that quadrature points coincide with the interpolation points for the ansatz functions $l_{p}$. Figure 10 illustrates an exemplary spectral element (for simplicity in 2D) with a polynomial solution and the volume as well as surface quadrature points. To simplify (21) we observe that the integrand in the first integral of (21) is a polynomial of order $2 P$ in any direction $\zeta_{1}, \zeta_{2}, \zeta_{3}$. Using the construction (19) and the exactness of the Gauss quadrature we get

$$
\begin{aligned}
\frac{\partial}{\partial t} \int_{E} \overline{\boldsymbol{U}}_{\boldsymbol{i}}^{\Delta x}(\boldsymbol{\xi}, t) \psi_{\boldsymbol{q}}(\boldsymbol{\xi}) \mathrm{d} \boldsymbol{\zeta} & =\frac{\partial}{\partial t} \sum_{\boldsymbol{p} \in \mathcal{P}} \int_{-1}^{1} \int_{-1}^{1} \int_{-1}^{1} \hat{\boldsymbol{U}}_{\boldsymbol{i}, \boldsymbol{p}}(t) \psi_{\boldsymbol{p}}(\boldsymbol{\zeta}) \psi_{\boldsymbol{q}}(\boldsymbol{\zeta}) \mathrm{d} \zeta_{1} \mathrm{~d} \zeta_{2} \mathrm{~d} \zeta_{3} \\
& =\omega_{q_{1}} \omega_{q_{2}} \omega_{q_{3}} \frac{\partial}{\partial t} \hat{\boldsymbol{U}}_{\boldsymbol{i}, \boldsymbol{q}}(t)
\end{aligned}
$$

To discretize the integral on the right hand side of (21) the nonlinear function $\mathcal{F}_{\boldsymbol{i}}^{1}\left(\overline{\boldsymbol{U}}_{\boldsymbol{i}}^{\Delta x}\right)$ is approximated by its interpolate, i.e.

$$
\int_{E} \mathcal{F}_{\boldsymbol{i}}^{1}\left(\overline{\boldsymbol{U}}_{\boldsymbol{i}}^{\Delta x}(\boldsymbol{\zeta})\right) \psi_{\boldsymbol{q}, \zeta_{1}}(\boldsymbol{\zeta}) \mathrm{d} \boldsymbol{\zeta} \approx \int_{E} I^{P}\left[\mathcal{F}_{\boldsymbol{i}}^{1}\left(\overline{\boldsymbol{U}}_{\boldsymbol{i}}^{\Delta x}\right)(\cdot, t)\right](\boldsymbol{\zeta}) \psi_{\boldsymbol{q}, \zeta_{1}}(\boldsymbol{\zeta}) \mathrm{d} \boldsymbol{\zeta}
$$

The integrand in the approximation is in $\mathcal{V}^{2 P-1}$. Thus we can exploit again the tensor structure and the exactness of the Gauss quadrature (22) to compute

$$
\int_{E} I^{P}\left[\mathcal{F}_{i}^{1}\left(\overline{\boldsymbol{U}}_{\boldsymbol{i}}^{\Delta x}\right)(\cdot, t)\right](\boldsymbol{\zeta}) \psi_{\boldsymbol{q}, \zeta_{1}}(\boldsymbol{\zeta}) \mathrm{d} \boldsymbol{\zeta}=\omega_{q_{2}} \omega_{q_{3}} \sum_{p=0}^{P} \omega_{p} l_{q_{1}}^{\prime}\left(\zeta^{p}\right) \mathcal{F}_{\boldsymbol{i}}^{1}\left(\overline{\boldsymbol{U}}_{\boldsymbol{i}}^{\Delta x}\left(\zeta^{p}, \zeta^{q_{2}}, \zeta^{q_{3}}, t\right)\right) .
$$

The other terms on the right hand side of (21) are approximated in the analogous way. It remains to consider the surface integrals in $(21)$. Let e.g. $k=k(\boldsymbol{i}, \boldsymbol{j})=+1$. Again we interpolate the nonlinear function $\mathcal{F}_{\boldsymbol{i}}^{*,+1}\left(\overline{\boldsymbol{U}}_{\boldsymbol{i}}^{\Delta x}\left(+1, \zeta_{2}, \zeta_{3}, t\right), \overline{\boldsymbol{U}}_{\boldsymbol{j}}^{\Delta x}\left(-1, \zeta_{2}, \zeta_{3}, t\right)\right)$ and consider the approximation

$$
\begin{aligned}
\int_{E_{+1}} \mathcal{F}_{i}^{*,+1}\left(\overline{\boldsymbol{U}}_{\boldsymbol{i}}^{\Delta x}\left(+1, \zeta_{2}, \zeta_{3}, t\right), \overline{\boldsymbol{U}}_{\boldsymbol{j}}^{\Delta x}\left(-1, \zeta_{2}, \zeta_{3}, t\right)\right) \psi_{\boldsymbol{q}}\left(+1, \zeta_{2}, \zeta_{3}\right) \mathrm{d} \zeta_{2} \mathrm{~d} \zeta_{3} \\
\quad \approx \int_{E_{+1}} I_{+1}^{P}\left[\mathcal{F}_{\boldsymbol{i}}^{*,+1}\left(\overline{\boldsymbol{U}}_{\boldsymbol{i}}^{\Delta x}(+1, \cdot, \cdot, t), \overline{\boldsymbol{U}}_{\boldsymbol{j}}^{\Delta x}(-1, \cdot, \cdot, t)\right)\right]\left(\zeta_{2}, \zeta_{3}\right) \psi_{\boldsymbol{q}}\left(+1, \zeta_{2}, \zeta_{3}\right) \mathrm{d} \zeta_{2} \mathrm{~d} \zeta_{3}
\end{aligned}
$$




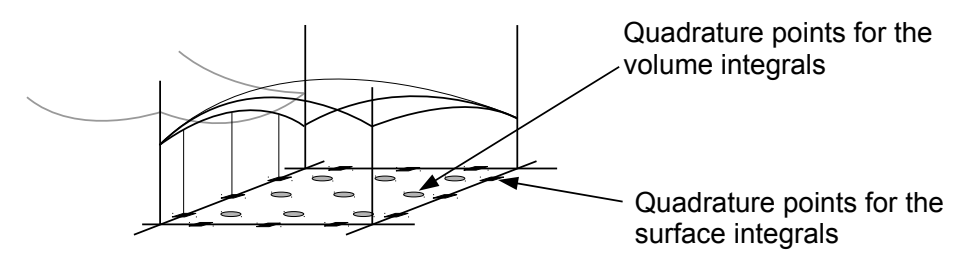

Figure 10. 2D schematic illustrating the polynomial solutions (degree $P=2$ ) of two neighboring spectral elements as well as the location of volume and surface quadrature points.

The integrand in the approximation is in $\mathcal{V}_{+1}^{2 P}$. Tensor structure and the exactness of the Gauss quadrature (22) lead to

$$
\begin{aligned}
\int_{E_{+1}} & I_{+1}^{P}\left[\mathcal{F}_{i}^{*,+1}\left(\overline{\boldsymbol{U}}_{\boldsymbol{i}}^{\Delta x}(+1, \cdot, \cdot, t), \overline{\boldsymbol{U}}_{\boldsymbol{j}}^{\Delta x}(-1, \cdot, \cdot, t)\right)\right]\left(\zeta_{2}, \zeta_{3}\right) \psi_{\boldsymbol{q}}\left(+1, \zeta_{2}, \zeta_{3}\right) \mathrm{d} \zeta_{2} \mathrm{~d} \zeta_{3} \\
& =\omega_{q_{2}} \omega_{q_{3}} l_{q_{1}}(+1) \mathcal{F}_{\boldsymbol{i}}^{*,+1}\left(\overline{\boldsymbol{U}}_{\boldsymbol{i}}^{\Delta x}\left(+1, \zeta^{q_{2}}, \zeta^{q_{3}}, t\right), \overline{\boldsymbol{U}}_{\boldsymbol{j}}^{\Delta x}\left(-1, \zeta^{q_{2}}, \zeta^{q_{3}}, t\right)\right) .
\end{aligned}
$$

If we put together the computations from (23), (25), and (26) we obtain the (semi discrete) DGSEM formulation as

$$
\begin{aligned}
\frac{\partial}{\partial t} \hat{\boldsymbol{U}}_{\boldsymbol{i}, \boldsymbol{q}}(t)= & -\frac{l_{q_{1}}(1)}{q_{1}} \mathcal{F}_{\boldsymbol{i}}^{*, 1}\left(\overline{\boldsymbol{U}}_{\boldsymbol{i}}^{\Delta x}\left(1, \zeta^{q_{2}}, \zeta^{q_{3}}, t\right), \overline{\boldsymbol{U}}_{\boldsymbol{i}+\boldsymbol{e}_{1}}^{\Delta x}\left(-1, \zeta^{q_{2}}, \zeta^{q_{3}}, t\right)\right) \\
& -\frac{l_{q_{1}}(-1)}{\omega_{q_{1}}} \mathcal{F}_{\boldsymbol{i}}^{*,-1}\left(\overline{\boldsymbol{U}}_{\boldsymbol{i}}^{\Delta x}\left(-1, \zeta^{q_{2}}, \zeta^{q_{3}}, t\right), \overline{\boldsymbol{U}}_{\boldsymbol{i}-\boldsymbol{e}_{1}}^{\Delta x}\left(1, \zeta^{q_{2}}, \zeta^{q_{3}}, t\right)\right) \\
& -\frac{l_{q_{2}}(1)}{\omega_{q_{2}}} \mathcal{F}_{i}^{*, 2}\left(\overline{\boldsymbol{U}}_{\boldsymbol{i}}^{\Delta x}\left(\zeta^{q_{1}}, 1, \zeta^{q_{3}}, t\right), \overline{\boldsymbol{U}}_{\boldsymbol{i}+\boldsymbol{e}_{2}}^{\Delta x}\left(\zeta^{q_{1}},-1, \zeta^{q_{3}}, t\right)\right) \\
& -\frac{l_{q_{2}}(-1)}{\omega_{q_{2}}} \mathcal{F}_{i}^{*,-2}\left(\overline{\boldsymbol{U}}_{\boldsymbol{i}}^{\Delta x}\left(\zeta^{q_{1}},-1, \zeta^{q_{3}}, t\right), \overline{\boldsymbol{U}}_{\boldsymbol{i}-\boldsymbol{e}_{2}}^{\Delta x}\left(\zeta^{q_{1}}, 1, \zeta^{q_{3}}, t\right)\right) \\
& -\frac{l_{q_{3}}(1)}{\omega_{q_{3}}} \mathcal{F}_{\boldsymbol{i}}^{*, 3}\left(\overline{\boldsymbol{U}}_{\boldsymbol{i}}^{\Delta x}\left(\zeta^{q_{1}}, \zeta^{q_{2}}, 1, t\right), \overline{\boldsymbol{U}}_{\boldsymbol{i}+\boldsymbol{e}_{3}}^{\Delta x}\left(\zeta^{q_{1}}, \zeta^{q_{2}},-1, t\right)\right) \\
& -\frac{l_{q_{2}}(-1)}{\omega_{q_{3}}} \mathcal{F}_{\boldsymbol{i}}^{*,-3}\left(\overline{\boldsymbol{U}}_{\boldsymbol{i}}^{\Delta x}\left(\zeta^{q_{1}}, \zeta^{q_{2}},-1, t\right), \overline{\boldsymbol{U}}_{\boldsymbol{i}-\boldsymbol{e}_{3}}^{\Delta x}\left(\zeta^{q_{1}}, \zeta^{q_{2}}, 1, t\right)\right) \\
& +\sum_{p=0}^{P} \frac{\omega_{p}}{\omega_{q_{1}}} l_{q_{1}}^{\prime}\left(\zeta^{p}\right) \mathcal{F}_{i}^{1}\left(\overline{\boldsymbol{U}}_{\boldsymbol{i}}^{\Delta x}\left(\zeta^{p}, \zeta^{q_{2}}, \zeta^{q_{3}}, t\right)\right) \\
& +\sum_{p=0}^{P} \frac{\omega_{p}}{\omega_{q_{2}}} l_{q_{2}}^{\prime}\left(\zeta^{p}\right) \mathcal{F}_{\boldsymbol{i}}^{2}\left(\overline{\boldsymbol{U}}_{\boldsymbol{i}}^{\Delta x}\left(\zeta^{q_{1}}, \zeta^{p}, \zeta^{q_{3}}, t\right)\right) \\
& +\sum_{p=0}^{P} \frac{\omega_{p}}{\omega_{q_{3}}} l_{q_{3}}^{\prime}\left(\zeta^{p}\right) \mathcal{F}_{i}^{3}\left(\overline{\boldsymbol{U}}_{\boldsymbol{i}}^{\Delta x}\left(\zeta^{q_{1}}, \zeta^{q_{2}}, \zeta^{p}, t\right)\right) .
\end{aligned}
$$

This scheme is used to solve the isothermal Euler equations (1) in conjunction with the van der Waals pressure function in the bulk phases (3), i.e. in liquid or vapor elements away from the interface, where a special procedure described in Section 4 is applied. The time integration for (27) relies on a fourth-order Runge-Kutta scheme.

\subsection{The Discontinuous Galerkin spectral element method for the 3D level-set transport}

The HMM approach used in this study relies on accurate geometrical information (position, curvature) of the liquid-vapor phase boundary $\Gamma(t)$, which necessitates a suitable tracking formalism. To obtain this information we use a level-set formulation: an additional function $\Phi: \Omega \times(0, T) \rightarrow \mathbb{R}$ is initialized as a signed distance function with respect to $\Gamma(0)$. It is then advected by a velocity field $\boldsymbol{s}_{\mathrm{PB}}: \Omega \times(0, T) \rightarrow \mathbb{R}^{d}$, to be defined. The 
advection equation for the level-set reads:

$$
\frac{\partial \Phi}{\partial t}+s_{\mathrm{PB}} \cdot \operatorname{grad}(\Phi)=0 \text { in } \Omega \times(0, T)
$$

Of course, this is only a conservation law as long as $\operatorname{div}\left(\boldsymbol{s}_{\mathrm{PB}}\right)=0$, which will not hold in our case. To discretize (28) also with the DGSEM scheme, equation (28) is re-cast as a conservation law with an additional right-hand-side containing the divergence of the level-set advection speed $s_{\mathrm{PB}}$ :

$$
\frac{\partial \Phi}{\partial t}+\operatorname{div}\left(\boldsymbol{s}_{\mathrm{PB}} \Phi\right)=\Phi \operatorname{div}\left(\boldsymbol{s}_{\mathrm{PB}}\right)
$$

Note that the divergence term can be readily obtained in the DGSEM by evaluating the necessary derivatives of the high-order ansatz polynomials.

The choice of the level-set advection speed $s_{\mathrm{PB}}$ is a crucial point in this study. Ideally it coincides with the local normal speed of the phase boundary $\Gamma(t)$ and is Lipschitz in $\Omega$. The (mesh-dependent) procedure to construct $s_{\mathrm{PB}}$ from the information obtained by the micro-scale solver is described in Section 4.1.2 below.

\section{TRANSFER OF INFORMATION BETWEEN MACRO-SCALE AND MICRO-SCALE SOLVER}

In the framework of Algorithm 1.1, information has to be communicated between the micro-scale and macroscale solvers. In the case of the DGSEM scheme, this is achieved in the following way: for the $n$th time-step $t^{n} \in[0, T)$ the approximation of the liquid-vapor interface $\Gamma\left(t^{n}\right)$ is provided by the zero level-set $\Phi_{h}^{n}=\{\boldsymbol{x} \in$ $\left.\Omega \mid \Phi_{h}\left(\boldsymbol{x}, t^{n}\right)=0\right\}$ whereby $\Phi_{h}$ is the approximate solution of (28). Based on this information, grid cells primarily occupied by liquid or vapor are identified by the respective sign of the integral average of the level-set variable. This way, one obtains a set of element faces that separate liquid cells from vapor cells and constitute a secondary liquid vapor interface $\Gamma_{h}^{n}$, which will be referred to as computational interface in the following.

\subsection{Transfer micro-scale $\rightarrow$ macro-scale}

\subsubsection{Numerical fluxes at the computational interface}

In the bulk phases away from the computational interface $\Gamma_{h}^{n}$ at time $t=t^{n}$, the DGSEM incorporates approximate Riemann solvers (the HLLC solver [6] in this study) to obtain the numerical fluxes $\boldsymbol{F}^{*}$. An approximate Riemann solution is also required at each quadrature point of the surface integral contribution for the computational interface as illustrated in figure 11. For these elements with faces that belong to the computational interface, the micro-scale solver is used. Let $\boldsymbol{U}_{\text {liq }}:=\boldsymbol{U}^{-}$and $\boldsymbol{U}_{\text {vap }}:=\boldsymbol{U}^{+}$be the respective states in the two different phases in some quadrature point (the normal $\boldsymbol{n}$ is pointing into the vapor domain). These states (to be precise the velocity components are projected to the direction normal to the face and written in Lagrangian coordinates) are the input for the micro-scale solver (together with the curvature). The micro-scale solver delivers for each quadrature point a liquid state $\boldsymbol{U}_{\text {liq }}^{*}$ and a vapor state $\boldsymbol{U}_{\text {vap }}^{*}$ (see Section 2). The DGSEM now uses the flux evaluations $\boldsymbol{F}^{*}=\boldsymbol{F}\left(\boldsymbol{U}_{\text {liq }}^{*}\right)$ for the computation on the liquid element and $\boldsymbol{F}^{*}=\boldsymbol{F}\left(\boldsymbol{U}_{\text {vap }}^{*}\right)$ for the computation on the vapor element (instead of $\left.\boldsymbol{F}^{*}\left(\boldsymbol{U}_{\text {liq }}, \boldsymbol{U}_{\text {vap }}\right)\right)$. This method of calculating two different fluxes for each phase results in a solution where variables such as the density remain discontinuous across the (computational) interface and the typical numerical smearing of discontinuities in

numerical schemes is avoided. For piecewise constant approximations the method is similar to the ghostfluid method $[7,16]$, where two different fluxes are obtained via additional ghost states near the interface.

\subsubsection{Construction of a velocity field for the level-set transport}

By the definition of $\Gamma_{h}^{n}$, the jump in the flow variables will remain (temporarily) fixed to the computational interface, and thus to the grid. In general, however, the interface is not stationary. Information on its dynamics is provided by the advection of the level-set, which is driven by the propagation speed of the phase boundary $s$ 


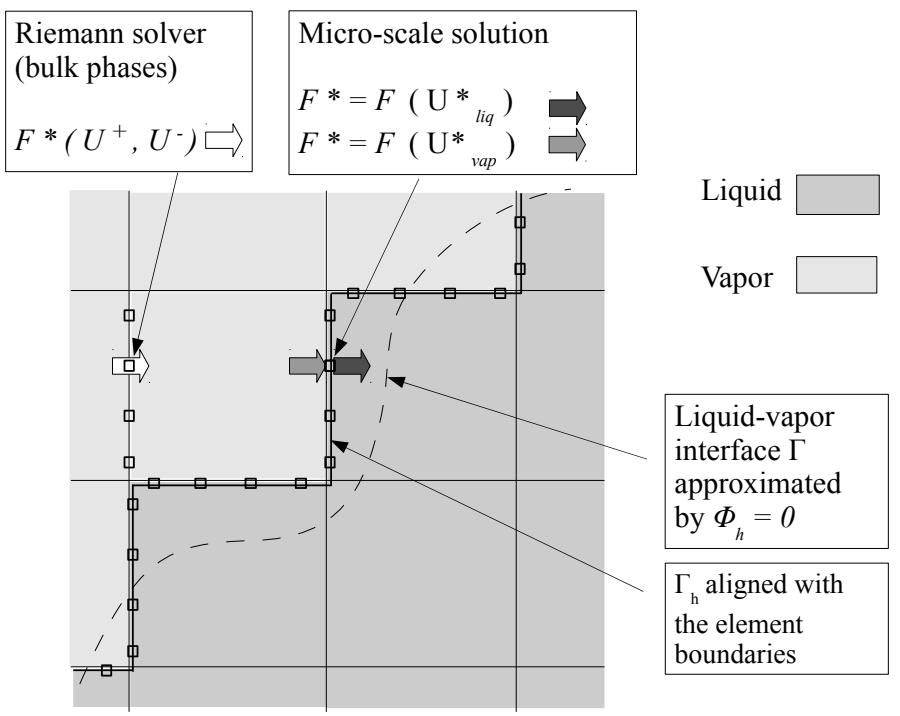

FIGURE 11. Schematic of a typical setting in the HMM approach, involving the liquid-vapor interface $\Gamma$, approximated by the zero level-set $\Phi_{h}^{n}$, the computational interface $\Gamma_{h}^{n}$ and the different ways to apply the numerical fluxes provided by either a standard Riemann solver (bulk phase) or the micro-scale solver at the computational interface.

given by the micro-scale solution (see (15)). Naturally, this movement leads to cases where the computational interface leaps from one cell boundary to the next, which means that a previously liquid cell instantaneously changes its definition from liquid to vapor (or vice versa). In such an event, all quantities inside this element are essentially unknown. In our approach, this cell is filled with an extrapolation of the states in neighboring elements of the same phase. We employ a method similar to the one described by Chertock and Kurganov [4].

Due to phase change, the fluid velocity may become discontinuous at the interface and as a result, the propagation speed of the phase boundary will in general be different from either of these velocities. In certain cases, the interface may even propagate in a direction opposite to the movement of the fluids it separates. An example is shown in figure 12 for a simulation with a high rate of evaporation. In the sharp interface approach considered here, this property applies also to the numerical representation of the physical phenomenon. Therefore, the only valid information available on propagation speed can be drawn from the micro-scale solver. The latter is applied at the computational interface, which in the general case does not coincide with the zero level-set. This discrepancy poses two different problems: the first is that these front propagation speeds are only applicable at the level-set zero per definition, it is therefore necessary to devise a procedure to transfer information from the computational interface to the physical interface approximated by $\Phi_{h}^{n}$. Furthermore, the level-set is defined in the entire domain, not just at the interface; in order to keep deviations from the shape of a signed distance function as small as possible, a velocity field is needed in the volume, not just at the interface. Both problems can be resolved by constructing an artificial velocity field for level-set transport in the volume. For practicality, this is only done in a neighborhood of one or more grid cells around $\Gamma_{h}^{n}$.

In view of the structure of the DGSEM scheme, it is clear that such a velocity field can easily be created by assigning one vector to each of the interpolation points for the polynomials in each spectral element (see figure 13). The available information from the micro-scale is located at the surface quadrature points. The first idea is to assume that each micro solver creates a linear advection in normal direction to the computational interface, thereby affecting only the volume interpolation points situated along a straight line through the surface quadrature point considered. The second is that the velocity vectors should always be aligned with the 


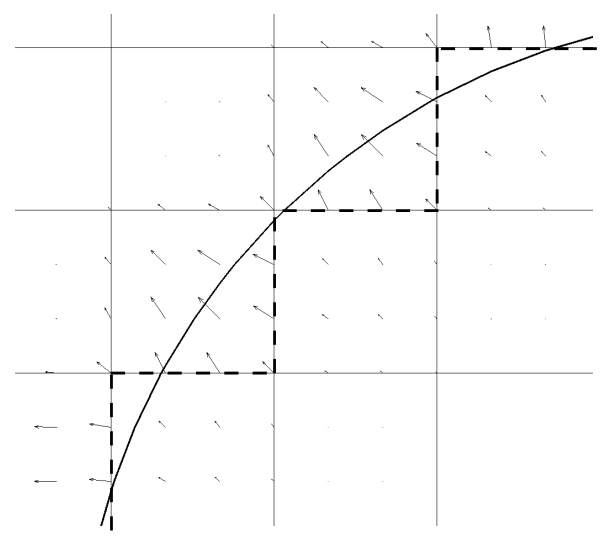

a)

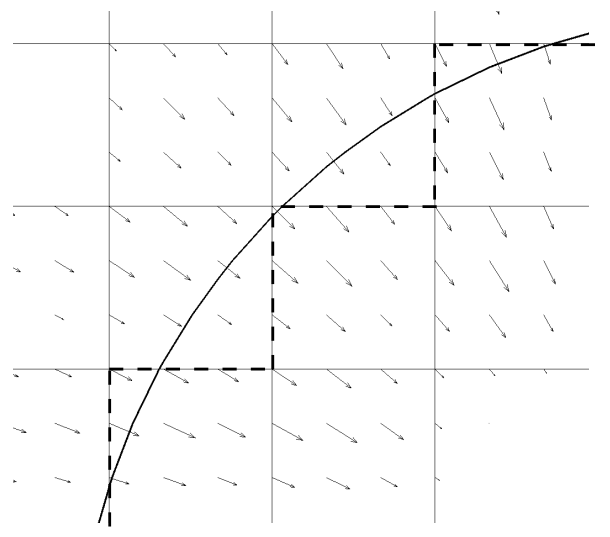

b)

FIgURE 12. Simulation result of a strongly evaporating droplet with surface tension. Continuous line: zero level-set contour $\Phi_{h}^{n}$, dashed line: computational interface $\Gamma_{h}^{n}$. a) vectors of the fluid velocity, b) vectors of the interface propagation speed $s_{\mathrm{PB}}$.

normal $\boldsymbol{n}$ of the level $\left\{\Phi_{h}\left(\cdot, t^{n}\right)=\right.$ const $\}$ that contains the interpolation point considered. It is given by

$$
\boldsymbol{n}\left(\boldsymbol{x}, t^{n}\right):=\left(\begin{array}{c}
n_{1}\left(\boldsymbol{x}, t^{n}\right) \\
n_{2}\left(\boldsymbol{x}, t^{n}\right)
\end{array}\right):=\frac{\operatorname{grad}\left(\Phi_{h}\left(\boldsymbol{x}, t^{n}\right)\right)}{\left|\operatorname{grad}\left(\Phi_{h}\left(\boldsymbol{x}, t^{n}\right)\right)\right|} .
$$

If only one element face coincides with a computational interface, the procedure is straightforward: figure 13 a) shows a propagation speed $s_{1}$ that is applied to the three interpolation points in normal direction, where it is rotated in the direction of the local normal vector $\boldsymbol{n}$. In the case where several element sides are part of the computational interface, several lines of linear transport intersect in one volume interpolation point. An example is illustrated in figure $13 \mathrm{~b}$ ) for two propagation speeds $\boldsymbol{s}_{1}$ and $\boldsymbol{s}_{2}$ that have to be superposed to obtain a resulting vector for the level-set propagation speed $s_{\mathrm{PB}}$. The magnitude of $s_{\mathrm{PB}}$ is then obtained by the expression

$$
\boldsymbol{s}_{\mathrm{PB}}=\frac{\boldsymbol{n} \cdot\left(\boldsymbol{s}_{1}+\boldsymbol{s}_{2}\right)}{\left|n_{1}\right|+\left|n_{2}\right|} \boldsymbol{n},
$$

which corresponds to a weighted average of $s_{1}$ and $s_{2}$. It further satisfies the following conditions for the limiting cases: (i) if $\left|s_{1}\right|=0$ and $\left|s_{2}\right| \neq 0$, then we obtain $s_{\mathrm{PB}}=s_{1}$ and vice versa, (ii) if $\left|s_{1}\right|=\left|s_{1}\right|$ and $\left|\boldsymbol{n}_{1}\right|=\left|\boldsymbol{n}_{2}\right|$, we obtain a magnitude of the resulting speed $\left|s_{\mathrm{PB}}\right|=\left|s_{1}\right|=\left|s_{2}\right|$. The latter property in particular will ensure that a physical interface propagating diagonally with respect to the grid does not deform despite the staircase shape of the computational interface. In a more general form, equation (31) reads

$$
\boldsymbol{s}_{\mathrm{PB}}=\frac{\boldsymbol{n} \cdot\left(\sum_{i=1}^{N_{F}} \boldsymbol{s}_{i}\right)}{\sum_{k=1}^{d}\left|n_{k}\right|} \boldsymbol{n}
$$

where $N_{F}$ is the number of element faces coinciding with the computational interface $\Gamma_{h}$. The applicable front propagation speed on the element face $i$ is denoted by $s_{i}$.

\subsection{Transfer macro-scale $\rightarrow$ micro-scale}

The Algorithm 1.1 includes the effect of surface tension. This necessitates two features to be incorporated in the numerical scheme: the first is the evaluation of the local curvature of the interface, the second is the application of the resulting surface force on the flow. In the literature, a common way to do the latter is to 


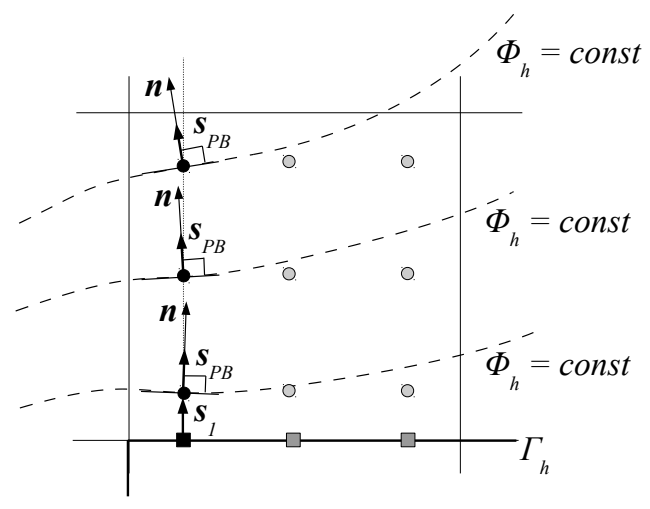

a)

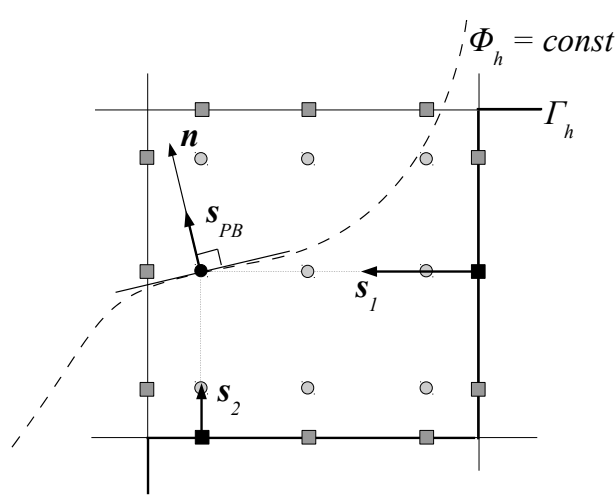

b)

FiguRE 13. Schematic of the procedure employed to calculate the velocity field $\boldsymbol{s}_{\mathrm{PB}}$ for level-set transport. a) situation in the case, where only one element face coincides with the computational interface. b) situation for two adjacent element faces coinciding with the computational interface.

replace the surface force by a distribution of a volume source term, e.g. the continuum surface force method of Brackbill and Kothe [2]. In the HMM approach of the present study, the effect of surface tension is entirely handled on the micro-scale (see section 2). This way, the surface tension force is exerted on the macro-scale through the fluxes at the computational interface. Inversely, it is necessary to provide the micro-scale with information on the mean curvature $\kappa$, which is obtained from the divergence of the level-set normal:

$$
\kappa=\operatorname{div}\left(\frac{\operatorname{grad}\left(\Phi_{h}\right)}{\left|\operatorname{grad}\left(\Phi_{h}\right)\right|}\right) .
$$

This quantity is only meaningful at the zero level-set, which in our approach does not coincide with the computational interface. Therefore, for each micro-solver, a location on the zero level-set that stands in a useful relation to the position of the surface integral quadrature point has to be found. One method yielding good results is for each surface quadrature point to search the zero level-set in the direction normal to the element face, as illustrated in figure 14. The value for the mean curvature $\kappa$ found at this point is used as initial data of the micro-scale solver.

\section{Numerical tests}

\subsection{A steady state droplet}

The first test case is a three-dimensional spherical droplet at static equilibrium in the domain $\Omega=(-1,1)^{3}$. The initial radius $r_{\text {ini }}$ of the droplet with its center at $(0,0,0)^{t}$ as well as the constant densities at the inside and at the outside of the droplet are chosen in such a way that the pressure difference resulting from surface tension is equal to the difference between the initial pressures (cf. Table 1 for a summary). In particular equation (7) is fulfilled for the van der Waals pressure (3) and constants in (4). A coarse grid of $30 \times 30 \times 30$ cells is used, the polynomial order is $N=2$ leading to a (formally) third-order scheme. The quadrature is of Gauss (Lobatto) type. The purpose of this test is to demonstrate the capability of our approach to conserve the steady-state even on grids, where the discrepancy between $\Gamma$ and the computational interface $\Gamma_{h}^{n}$ is significant. Figure 15 shows a) the zero level-set as well as b) the computational interface (represented by a density iso-surface) at the 100th time-step which corresponds to $T=0.166$. The density contour clearly reveals the pronounced staircase shape of the computational interface $\Gamma_{h}^{100}$, which is aligned with the grid. Clearly, the approximation of the spherical 


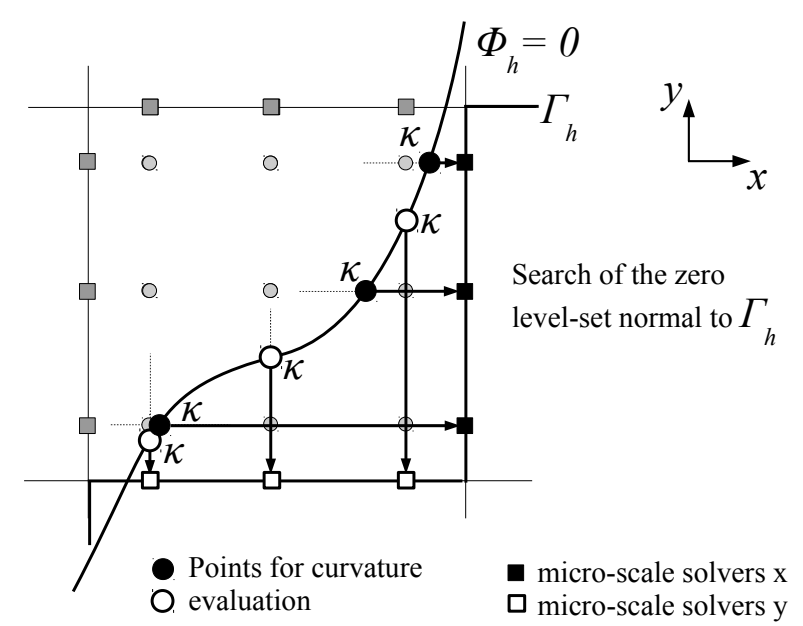

FiguRE 14. Schematic of the procedure used to evaluate the mean curvature $\kappa$ inside a spectral element.

\begin{tabular}{|l|l|c|c|}
\hline Initial droplet radius & Surface tension coeff. & Inner state & Outer state \\
\hline$r_{\text {ini }}=0.4$ & $\gamma=0.0025$ & $\varrho=1.802747026 \quad|\boldsymbol{v}|=0.0$ & $\varrho=0.316842926 \quad|\boldsymbol{v}|=0.0$ \\
\hline
\end{tabular}

TABLE 1. Initial conditions for a droplet in static equilibrium.

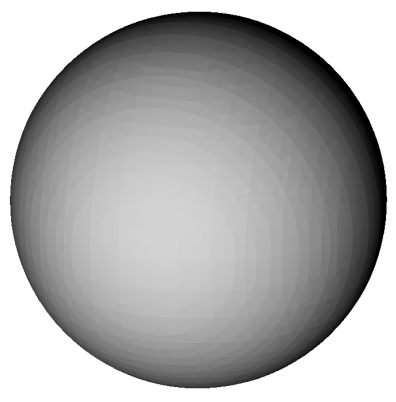

a)

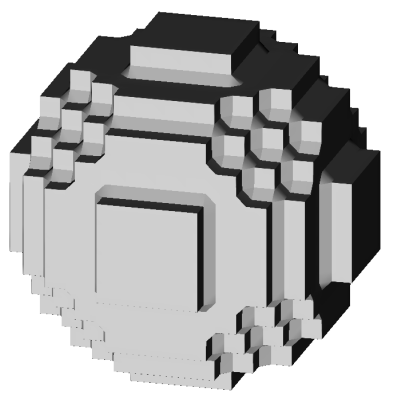

b)

FIGURE 15. Results for the steady state droplet test after 100 iterations. a) iso surface of the zero level-set $\Phi_{h}^{100}, \mathrm{~b}$ ) iso-surface of the density, which is equivalent to the computational interface $\Gamma_{h}^{100}$, apart from interpolation artifacts due to visualization.

droplet surface with $\Phi_{h}^{100}$, based on polynomial ansatz functions is vastly superior to the approximation by $\Gamma_{h}^{100}$. The smooth spherical shape is retained in the simulation result, showing that even on coarse grids the steady state is well conserved. Besides the visually good behavior, there are perturbations in the velocity field of order $10^{-4}$ which does not decrease within the short time span considered here.

Figure 16 shows pressure fields from the symmetry plane for two simulations. Part a) shows the result using the HMM approach including surface tension, part b) a result obtained with the surface tension model deactivated. The purpose of the latter test is to demonstrate that the effect of surface tension is not negligible 


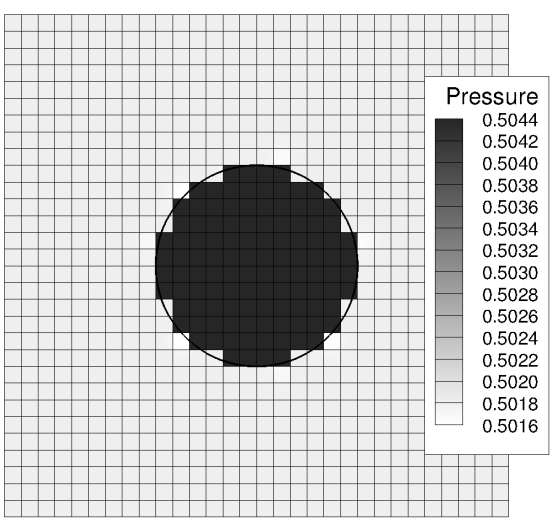

a)

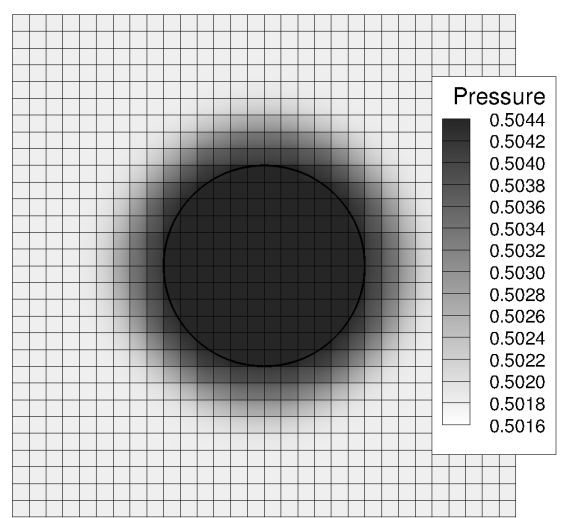

b)

FIGURE 16. Results for the steady state droplet test after 100 time-steps. Pressure fields on the symmetry plane, overlaid with the zero level-set (continuous black line). a) with surface tension model $(\gamma=0.0025)$, b) without surface tension model $(\gamma=0)$ for comparison.

\begin{tabular}{|l|l|c|c|}
\hline Initial bubble radius & Surface tension coefficient & Inner state & Outer state \\
\hline$r_{\text {ini }}=0.42$ & $\gamma=0.0025$ & $\varrho=0.3 \quad|\boldsymbol{v}|=0.0$ & $\varrho=1.828 \quad|\boldsymbol{v}|=0.0$ \\
\hline
\end{tabular}

TABLE 2. Initial conditions unsteady bubble test case.

under the chosen conditions. In each case, the zero level-set is added as a continuous black line. In the result using the surface tension model, the pressure jump is kept sharp, fluctuations along the computational interface are very minor. This shows that the curvature information from the level-set is accurate enough to retain the state of equilibrium. The quality of the result is underlined by the simulation without surface tension, where the equilibrium is not satisfied. This leads to a visible pressure wave forming at the interface, illustrating that large errors in (or as in case (b) the absence of) surface tension modeling would quickly affect the equilibrium state. Note that for very long end times $T$, the equilibrium in case (a) could still be perturbed as errors accumulate and lead to parasitic currents [10].

\subsection{An unsteady bubble}

The second test is a simulation of a spherical vapor bubble under conditions that lead to a rapid diameter decrease that is governed both by phase change and surface tension. This test demonstrates the capability of the HMM approach and in particular the geometric concepts from 4.1.2 to treat dynamical phase boundaries. The setting is as in the previous example and the initial states are detailed in Table 2. The pressure function is described in (3), (4). The computational domain is discretized with a grid of $30 \times 30 \times 30$ hexahedral elements. The polynomial order is again $N=2$. The bubble is initialized at the center of the domain located at $\boldsymbol{x}=(0,0,0)^{t}$. Figure 17 a) shows the pressure field on a symmetry plane of the domain together with the contour of the the bubble surface at $t=0.2$. Despite the deliberately coarse grid ( 8 cells across the bubble diameter), the pressure waves created by the initial jump at the staircase-shaped computational interface are smooth and circular. Likewise, the contour of the interface (or the zero level-set) retains its spherical shape as shown in more detail in figure $17 \mathrm{~b}$ ). In the same image, vectors of the level-set advection velocity $\boldsymbol{s}_{\mathrm{PB}}$ are shown. These vectors have the desired orientation normal to the interface and their magnitude is uniform regardless of their location relative to the computational interface. Note, however, that this observation only applies to a neighborhood of two grid elements with respect to the computational interface. Figures 18 a), 


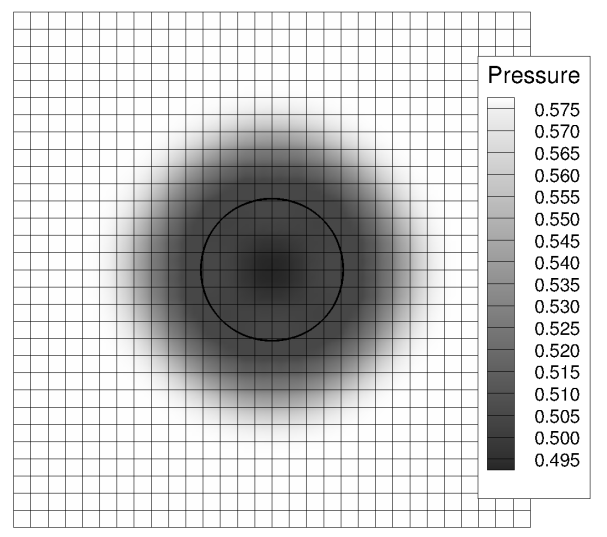

a)

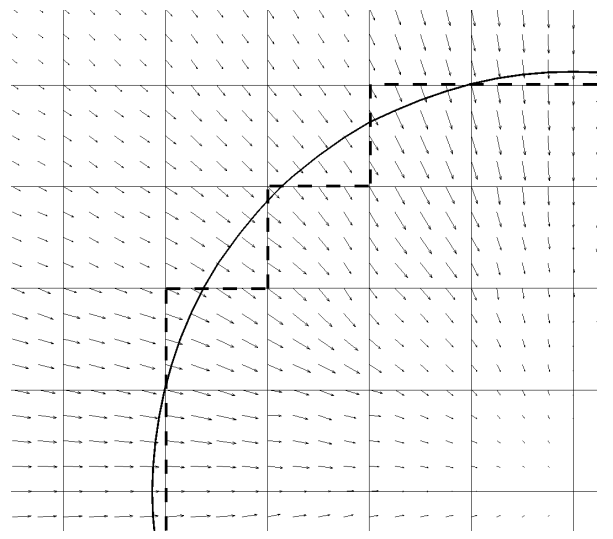

b)

Figure 17. Results for the unsteady bubble test at $t=0.2$. a) Pressure field on the symmetry plane, overlaid with the zero level-set (continuous line). b) level-set advection velocity field with the zero level-set (continuous line) and the computational interface (dashed line).

b), c) show a quantitative comparison between Algorithm 1.1 and a well-resolved 1D simulation in spherical coordinates (cf. [18] for details of the code) as reference solution. In view of the low resolution of the 3D results, agreement between both results is still fairly good. In particular, the discontinuities in the pressure (due to surface tension) and in the velocity (due to phase change) are clearly visible and well reproduced in the case of the pressure. For the velocity result, the jump is less pronounced in the 3D result compared to the 1D calculation.

As a quantity that depends on the interaction of all components involved, the bubble diameter temporal evolution is shown in figure 19. Again, we compare the well-resolved 1D simulation with the 3D HMM result on a very coarse grid. For the time interval considered $(T=0.4)$, there is good agreement between both results. Longer simulation times have not been considered as waves begin to interact with the domain boundaries, where a direct comparison between both results is no longer possible. Furthermore, in the early simulations shown here, re-initialization in order to keep $\Phi_{h}$ a distance function is not yet available, leading to a deterioration of the smoothness of the level-set variable and ultimately inaccurate curvature information. 


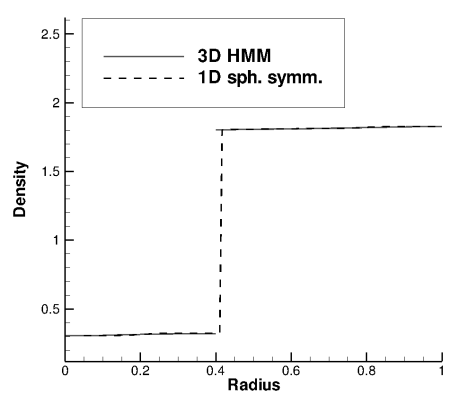

a)

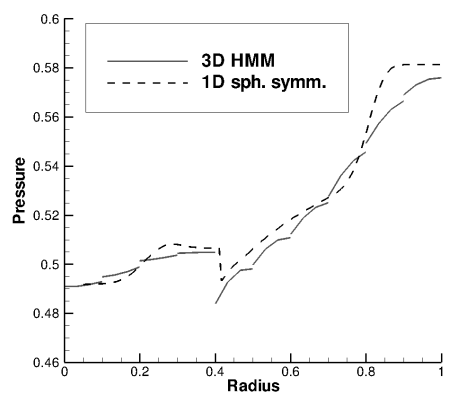

b)

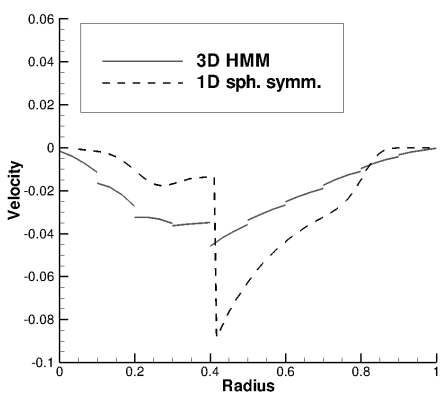

c)

FiguRE 18. Results for the unsteady bubble test at $t=0.2$. Comparison of the reference simulation in 1D assuming spherical symmetry and the 3D solution using the HMM approach. Plots of a) density, b) pressure and c) velocity, each plotted over the radius (1D) or along a line in radial direction $(3 \mathrm{D})$.

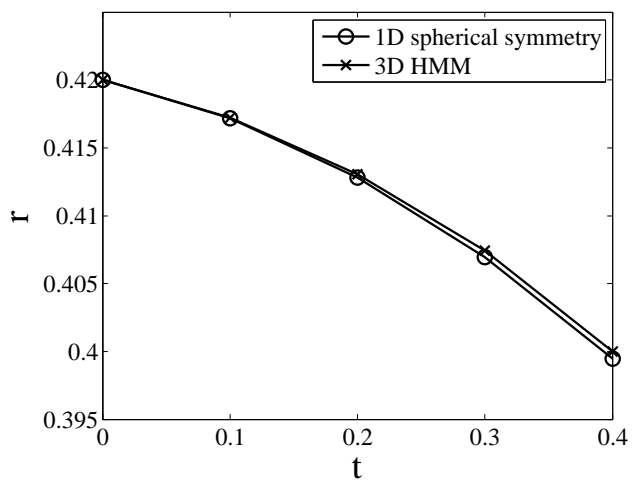

Figure 19. Evolution of the bubble radius $r$ over time $t$. Comparison of the reference simulation in 1D assuming spherical symmetry and the 3D solution using the HMM approach. 


\section{REFERENCES}

[1] S. Benzoni-Gavage and H. Freistühler. Effects of surface tension on the stability of dynamical liquid-vapor interfaces. Arch. Ration. Mech. Anal., 174(1):111-150, 2004.

[2] J.U. Brackbill, D.B. Kothe, and C. Zemach. A continuum method for modeling surface tension. Journal of computational physics, 100(2):335-354, 1992.

[3] F. Caro, F. Coquel, D. Jamet, and S. Kokh. A simple finite-volume method for compressible isothermal two-phase flows simulation. Int. J. Finite Vol., 3(1):37, 2006.

[4] A. Chertock and A. Kurganov. A simple eulerian finite-volume method for compressible fluids in domains with moving boundaries. Communications in Mathematical Sciences, 6(3):531-556, 2008.

[5] A. Dressel and C. Rohde. A finite-volume approach to liquid-vapour fluids with phase transition. In Finite volumes for complex applications $V$, pages 53-68. ISTE, London, 2008.

[6] E.F. Toro, M. Spruce, and W. Speares. Restoration of the contact surface in the hll-riemann solver. Shock waves, 4(1):25-34, 1994.

[7] R.P. Fedkiw, T. Aslam, B. Merriman, and S. Osher. A non-oscillatory Eulerian approach to interfaces in multimaterial flows (the ghost fluid method). J. Comput. Phys., 152(2):457-492, 1999.

[8] Edwige Godlewski and Nicolas Seguin. The Riemann problem for a simple model of phase transition. Commun. Math. Sci., $4(1): 227-247,2006$.

[9] M.E. Gurtin. On a theory of phase transitions with interfacial energy. Arch. Rational Mech. Anal., 87(3):187-212, 1985.

[10] D.J.E. Harvie, M.R. Davidson, and M. Rudman. An analysis of parasitic current generation in volume of fluid simulations. Applied mathematical modelling, 30(10):1056-1066, 2006.

[11] F. Jaegle and V. Schleper. Exact and approximate riemann solvers at phase boundaries. Preprint, 2012. http://www.ians. uni-stuttgart.de/am/Schleper/Preprints/Riemann.pdf.

[12] F. Kissling and C. Rohde. The computation of nonclassical shock waves with a heterogeneous multiscale method. Netw. Heterog. Media, 5(3):661-674, 2010.

[13] D.A. Kopriva. Spectral Element Methods. Implementing Spectral Methods for Partial Differential Equations, pages 293-354, 2009.

[14] David A. Kopriva and Gregor Gassner. On the Quadrature and Weak Form Choices in Collocation Type Discontinuous Galerkin Spectral Element Methods. J. Sci. Comput., 44:136-155, 2010.

[15] T.P. Liu. The Riemann problem for general systems of conservation laws. J. Differential Equations, 18:218-234, 1975.

[16] C. Merkle and C. Rohde. The sharp-interface approach for fluids with phase change: Riemann problems and ghost fluid techniques. M2AN Math. Model. Numer. Anal., 41(6):1089-1123, 2007.

[17] S. Müller and A. Voß. The Riemann problem for the Euler equations with nonconvex and nonsmooth equation of state: construction of wave curves. SIAM J. Sci. Comput., 28(2):651-681 (electronic), 2006.

[18] C. Rohde and C. Zeiler. A multi-scale algorithm for compressible liquid-vapour flow with surface tension. In preparation.

[19] Y. Sun and B. Engquist. Heterogeneous multiscale methods for interface tracking of combustion fronts. Multiscale Model. Simul., 5(2):532-563, 2006.

[20] V.A. Solonnikov and A. Tani. Evolution free boundary problem for equations of motion of viscous compressible barotropic liquid. In The Navier-Stokes equations II-theory and numerical methods (Oberwolfach, 1991), volume 1530 of Lecture Notes in Math., pages 30-55. Springer, Berlin, 1992.

[21] W. E, B. Engquist, X. Li, W. Ren, and E. Vanden-Eijnden. Heterogeneous multiscale methods: a review. Commun. Comput. Phys., 2(3):367-450, 2007. 